\title{
Multiplicity-free Representations and Visible Actions on Complex Manifolds ${ }^{\dagger}$
}

By

\author{
Toshiyuki KOBAYASHI*
}

\section{Contents}

$\S 1 . \quad$ Introduction

§1.1. Classical analysis from multiplicity-free representations

§1.2. Multiplicity-free representations - some known examples

$\S 1.3$. Propagation of multiplicity-free property and visible actions

$\S 1.4$. Multiplicity-free representations and explicit decomposition formulas

§1.5. Multiplicity-free representations - definition

$\S 2$. Multiplicity-Free Theorem - General Framework

$\S 2.1$. Holomorphic bundles and anti-holomorphic maps

$\S 2.2$. Multiplicity-free theorem - line bundle case

$\S 2.3$. Geometry on the base space $D$

$\S 2.4$. Multiplicity-free theorem — vector bundle case

$\S 3$. Visible Actions on Complex Manifolds

$\S 3.1$. Previsible and visible actions on complex manifolds

§3.2. Infinitesimal characterization for visible actions

$\S 3.3 . \quad$ Anti-holomorphic map

Communicated by T. Kawai. Received December 28, 2004.

Partly supported by Grand-in-Aid for Exploratory Research 16654014, Japan Society of the Promotion of Science.

2000 Mathematics Subject Classification(s): Primary 22E46; Secondary 32A37, 05E15, $20 \mathrm{G} 05$.

Key words: multiplicity-free representation, branching law, visible action, semisimple Lie group, Hermitian symmetric space, flag variety, totally real submanifold

${ }^{\dagger}$ This article is an invited contribution to a special issue of Publications of RIMS commemorating the fortieth anniversary of the founding of the Research Institute for Mathematical Sciences.

*RIMS, Kyoto University, Kyoto 606-8502, Japan.

(C) 2005 Research Institute for Mathematical Sciences, Kyoto University. All rights reserved. 
$\S 4$. Coisotropic Actions, Polar Actions, and Visible Actions

$\S 4.1$. Coisotropic actions on symplectic manifolds

§4.2. Polar actions on Riemannian manifolds

§4.3. Actions on Kähler manifolds

$\S 4.4$. Visible actions and multiplicity-free representations

$\S 5$. Some Examples of Visible Actions

§5.1. Examples of visible actions 1 - abelian case

$\S 5.2$. Examples of visible actions 2 - group case

$\S 5.3$. Examples of visible actions 3 - complex symmetric case

§5.4. Examples of visible actions 4 - Hermitian symmetric case

$\S 5.5$. Examples of visible actions 5 - non-symmetric case

$\S 5.6$. Examples of visible actions 6 - linear multiplicity-free space

§5.7. Examples of visible actions 7 - spherical nilpotent varieties

$\S 5.8$. Examples of visible actions 8 - Stein extension of a Riemannian symmetric space

$\S 6$. Multiplicity-Free Representations — Finite Dimensional Examples

$\S 6.1$. Multiplicity-free tensor product representations

§6.2. Parabolic subalgebras with abelian nilradicals

$\S 6.3$. Multiplicity-free tensor product representations of $\mathfrak{g l}(n, \mathbb{C})$

§7. Multiplicity-Free Representations - Compact Examples

§7.1. Regular representation on symmetric spaces - scalar case

$\S 7.2$. Regular representation on symmetric spaces — vector bundle case

§7.3. Some finite dimensional applications

$\S 8$. Multiplicity-Free Representations - Infinite Dimensional Examples

$\S 8.1$. Unitary highest weight representations

§8.2. Restriction to a unipotent subgroup $N$

$\S 8.3$. Restriction to a symmetric subgroup

$\S 8.4$. Boundedness and uniform boundedness of multiplicities

$\S 8.5$. A generalization of Hua-Kostant-Schmid formula

§8.6. Multiplicity-free tensor product representations

$\S 9$. The Orbit Method

References 


\section{$\S 1 . \quad$ Introduction}

\section{§1.1. Classical analysis from multiplicity-free representations}

Multiplicity-free representations appear in various contexts of mathematics, though we may not be aware of even the fact that the representation is there.

For example, Taylor series expansion $f(z)=\sum_{\alpha \in \mathbb{N}^{n}} a_{\alpha} z^{\alpha}$ is built on the fact that each monomial $z^{\alpha}=z_{1}^{\alpha_{1}} \cdots z_{n}^{\alpha_{n}}$ appears only once. We can interpret this fact as the multiplicity-free property of the representation of the $n$-torus $\mathbb{T}^{n}$ on $\mathcal{O}\left(\mathbb{C}^{n}\right)$. Likewise, one can observe that Fourier transform is built on the multiplicity-free property of the regular representation of the abelian Lie group $\mathbb{R}^{n}$ on $L^{2}\left(\mathbb{R}^{n}\right)$.

In general, irreducible decomposition of a representation has an inevitable ambiguity if the multiplicity of irreducibles is greater than one. A distinguishing feature of multiplicity-free representation is that the representation space decomposes canonically into irreducibles. This in turn leads to a natural expansion of a function in the case the representation space consists of functions. If the multiplicity of $z^{\alpha}$ were larger than one in the Taylor expansion, such "expansion" would not be a powerful tool in analysis.

One of the advantages of the canonical irreducible decomposition for multiplicity-free representation is that such a decomposition diagonalizes any operator commuting with the group action. For example, the Laplacian $\Delta$ on the unit sphere $S^{n}$ commutes with the action of the orthogonal group $O(n+1)$. Since $O(n+1)$ acts on $L^{2}\left(S^{n}\right)$ with multiplicity free, the Laplacian is diagonalized, namely, each irreducible subspace becomes automatically an eigenspace of $\Delta$. This viewpoint yields a group-theoretic approach to the classical theory of spherical harmonics and more generally the analysis on Riemannian symmetric spaces.

Multiplicity-free properties also play a useful role in representation theory itself. The close interaction with invariant theory ([20]), construction of Gelfand-Tsetlin basis, study of degenerate principal series representations by means of multiplicity-free branching laws $([21,24,30,48$, etc.]) are a part of such successful applications.

\section{$\S 1.2$. Multiplicity-free representations - some known examples}

Over several decades, numerous multiplicity-free theorems have been found in various contexts including

- the Peter-Weyl theorem (Fact 28), 
- the Cartan-Helgason theorem on compact symmetric spaces (Fact 29),

- branching laws for $G L_{n} \downarrow G L_{n-1}$ and $O_{n} \downarrow O_{n-1}$ (Fact 31 and 32),

- Clebsch-Gordan formula,

- Pieri's law (Example 6.1.1),

- $G L_{m}-G L_{n}$ duality (Example 5.6.1),

- Plancherel formula for Riemannian symmetric spaces (Corollary 22),

- Gelfand-Graev-Vershik canonical representations (Example 8.3.3),

- Hua-Kostant-Schmid $K$-type formula for holomorphic discrete series representations (see Theorem 39 for its generalization),

- Kac's examples of linear multiplicity-free spaces (Theorem 19),

- Panyushev's classification of spherical nilpotent orbits (Theorem 20),

- Stembridge's classification of multiplicity-free tensor product representations of $G L_{n}$ (Theorem 27),

etc.

Accordingly, various techniques can be applied in each case. For example,

1) One can look for an open orbit of a Borel subgroup.

2) One can apply the Littlewood-Richardson rules and variants for the classical groups $S O(n, \mathbb{C}), S p(n, \mathbb{C})$.

3) One can use computational combinatorics.

4) One can employ the commutativity of the Hecke algebra.

5) One can apply Schur-Weyl duality and Howe duality.

However, no single known-method seems to cover all of the above multiplicity-free representations - not only in the finite dimensional case, but also in the infinite dimensional case for which the irreducible decomposition may involve continuous and discrete spectrum. 


\section{$\S 1.3$. Propagation of multiplicity-free property and visible actions}

The aim of this article is to present a simple principle based on complex geometry that explains various kinds of multiplicity-free representation. This paper is concerned with non-standard geometric perspectives of multiplicityfree representations in both finite and infinite dimensional cases, and in particular provides new proofs of classical multiplicity-free theorems in various contexts, along with a discussion of the complex geometry where a totally real submanifold meets generic orbits of a group (which we shall call 'previsible action' in Definition 3.1.1). Not only classical theorems but also a number of 'new' multiplicity-free theorems are naturally found from our machinery, most of which we supply a (at least, sketch of) proof here.

Our main machinery is an abstract multiplicity-free theorem for the sections of equivariant holomorphic vector bundles, and its most general form is stated in Theorem 2 in Section 2. The idea goes back to Gelfand's beautiful paper [13] which proved that the regular representation on a Riemannian symmetric space is multiplicity-free by showing the Hecke algebra is commutative. We then also extend Faraut-Thomas's work [12] on complex manifolds with anti-holomorphic involution.

Loosely speaking, our multiplicity-free results are built on the geometry of holomorphic vector bundles:

(1.3.1) Multiplicity-free action on the fiber,

(1.3.2) Visible action on the base space,

together with certain compatible conditions (see Theorem 2 for precision).

Here, the notion of (strongly) visible actions on a complex manifold is introduced in Definitions 3.1.1 and 3.3.1 in Section 3, and we shall elucidate this notion with various examples in Section 5 .

Putting a special emphasis on the condition (1.3.1), we may regard Theorem 2 as a propagation theorem of multiplicity-free property from a smaller group acting on the fiber with multiplicity-free assumption to the whole group acting on the space of sections. It might be a good contrast that there have been well-developed theory on a 'propagation of unitarity', including the Mackey theory of unitarily induced representations and the ZuckermanVogan-Wallach theorem of the unitarizability of cohomologically induced representations $([71,72])$. Unlike unitarity, the multiplicity-free property is not preserved in general for induced representations. In other words, a quite strong assumption should be made in order to establish a propagation theorem of 
multiplicity-free property. In this respect, the condition (1.3.2) (visible action) plays a key role in our case.

In Section 4, visible actions for complex manifolds are discussed in the broader context of symplectic geometry and Riemannian geometry. For this, we shall also discuss some relevant notions, namely, coisotropic actions on symplectic manifolds (Guillemin and Sternberg [15], Huckleberry and Wurzbacher $[22]$ ), and polar actions on Riemannian manifolds (Podestà and Thorbergsson $[57,58])$.

It is important to know that the condition defining visible action is local, and thus the same geometry often gives rise to multiplicity-free theorems simultaneously in both finite and infinite dimensional representations. As a simple example of this nature, we note that the tensor product representation of two irreducible (finite dimensional) representations of $S U(2)$ is multiplicity-free, and so is that of two discrete series representations of $S L(2, \mathbb{R})$. The former is explained by that the diagonal action of $S U(2)$ on $\mathbb{P}^{1} \mathbb{C} \times \mathbb{P}^{1} \mathbb{C}$ is visible, and the latter replaced by $S L(2, \mathbb{R})$. This nature will be formulated, in particular for semisimple symmetric pairs in Sections 5,6 and 8 .

\section{$\S 1.4$. Multiplicity-free representations and explicit decomposition formulas}

Multiplicity-free representations are a very special class of representations, for which one could expect a simple and detailed study. If a representation is known to be multiplicity-free, one may be tempted to find its irreducible decomposition explicitly. Since our geometric machinery has produced a number of new multiplicity-free theorems, it seems promising to pursue a deeper study of those multiplicity-free representations.

In fact, an extensive study of finding explicit formulas has been developed in recent years. It includes:

1) Okada-Krattenthaler's identities for classical group characters $([46,53])$.

2) A generalization of Hua-Kostant-Schmid formula to semisimple symmetric pairs [32], see also $§ 8.5)$.

We can tell these representations are multiplicity-free without a computation of explicit formulas by our machinery (Theorem 2), as we see Theorem 26 and 27 for the former (the finite dimensional case) and Theorem 34 for the latter (the infinite dimensional case).

These examples are algebraic formulas. Further study from analytic aspect (e.g. Parseval-Plancherel type theorem) would be also interesting. There 
has been recent progress in this direction, including Ben Saïd ([3]), ØrstedZhang [55], van Dijk-Hille [9] and Neretin [52]. In this connection, Zhang ([76]) has made a remarkable observation that the irreducible decomposition of multiplicity-free tensor product representations arisen from our framework (see Theorem 40) contains 'new' spherical irreducible unitary representations of some non-split reductive Lie groups as discrete spectrum (see Barbasch [2] for the current status on the classification of spherical unitary dual).

\section{§1.5. Multiplicity-free representations — definition}

Much of the literature of multiplicity-free representations deals with algebraic representations. However, our main interest is in both finite and infinite dimensional representations. This subsection reviews and fixes the definition of multiplicity-freeness for algebraic representations, unitary representations, and more generally, continuous representations.

Algebraic representations are called multiplicity-free if each irreducible representation occurs at most once. The same definition makes sense also for the unitary representations that are discretely decomposable into irreducible representations. More generally, the concept of multiplicity-free representations can be defined for unitary representations as follows:

Definition 1.5.1. Let $\pi$ be a unitary representation of a group $H$ on a (separable) Hilbert space $\mathcal{H}$. We write $\operatorname{End}_{H}(\mathcal{H})$ for the ring of continuous endomorphisms commuting with $H$. Then, we say $(\pi, \mathcal{H})$ is multiplicity-free if the ring $\operatorname{End}_{H}(\mathcal{H})$ is commutative.

Definition 1.5.1 implies, in particular, that the multiplicity of discrete spectrum is free, namely, the dimension of $\operatorname{Hom}_{H}(\tau, \pi)$ is at most one for any irreducible unitary representation $\tau$ of $H$, where $\operatorname{Hom}_{H}(\tau, \pi)$ denotes the space of continuous $H$-intertwining operators. However, Definition 1.5.1 covers not merely discrete spectrum but also continuous spectrum. To see this, suppose that the von Neumann algebra generated by $\pi(g)(g \in H)$ is of type I (for example, this is the case if $H$ is a reductive Lie group or a nilpotent Lie group). Then, the unitary representation $\pi$ decomposes into the direct integral of irreducible unitary representations in a unique way (up to unitary equivalence):

$$
\pi \simeq \int_{\widehat{H}}^{\oplus} m_{\pi}(\tau) \tau d \mu(\tau),
$$

where $\widehat{H}$ is the unitary dual of $H$ (the set of irreducible unitary representations of $H$ up to equivalence), $\mu$ is a Borel measure on $\widehat{H}$, and $m_{\pi}: \widehat{H} \rightarrow \mathbb{N} \cup\{\infty\}$ 
is the multiplicity. Then, the terminology "multiplicity-free" is justified by the following well-known Proposition, which is a consequence of Schur's lemma for unitary representations:

Proposition 1.5.2. The following two conditions are equivalent:

i) $\operatorname{End}_{H}(\mathcal{H})$ is commutative.

ii) $m_{\pi}(\tau) \leq 1$ for almost all $\tau \in \widehat{H}$ with respect to the measure $\mu$.

Later, our primary objects of study are representations realized on Fréchet spaces (e.g., the space of holomorphic functions). For such representations, we need to extend the concept of multiplicity-free representations as follows:

Definition 1.5.3. Suppose $\varpi$ is a continuous representation of $H$ on a topological vector space $W$. We say that $(\varpi, W)$ is multiplicity-free if any unitary representation $(\pi, \mathcal{H})$ with the property $(1.1)$ is multiplicity-free.

(1.1) There exists an injective and continuous $H$-intertwining operator $\mathcal{H} \hookrightarrow W$.

The following proposition is an immediate consequence of Definition 1.5.3.

Proposition 1.5.4. If $(\varpi, W)$ is multiplicity-free in the sense of Definition 1.5.3, then

$$
\operatorname{dim} \operatorname{Hom}_{H}(\tau, \varpi) \leq 1
$$

for any irreducible unitary representation $\tau$ of $H$.

Further, Definition 1.5.3 coincides with Definition 1.5.1 if $\varpi$ itself is unitary.

Proposition 1.5.5. If $(\varpi, W)$ is a unitary representation, then Definition 1.5.3 is equivalent to Definition 1.5.1.

Proof. In the setting (1.1), the closure $\overline{\mathcal{H}}$ in $W$ and its orthogonal complementary subspace $\mathcal{H}^{\perp}$ are both $H$-invariant. By using the direct sum decomposition $W=\overline{\mathcal{H}} \oplus \mathcal{H}^{\perp}$, we have naturally an embedding

$$
\operatorname{End}_{H}(\mathcal{H}) \hookrightarrow \operatorname{End}_{H}(W) .
$$

Hence, if $\operatorname{End}_{H}(W)$ is commutative then $\operatorname{End}_{H}(\mathcal{H})$ is commutative. This is what we wanted to prove. 
Remark 1.5.6. Multiple of non-unitary representations are out of scope in Definition 1.5.3. However, we can still discuss multiplicity-freeness of algebraic (or holomorphic) representations of complex reductive groups in the framework of Definition 1.5.3 by applying Weyl's unitary trick.

\section{§2. Multiplicity-Free Theorem - General Framework}

\section{§2.1. Holomorphic bundles and anti-holomorphic maps}

Let $\mathcal{V} \rightarrow D$ be a holomorphic vector bundle over a connected complex manifold $D$, associated to a finite dimensional representation $(\mu, V)$ of a Lie group $K$ and to a principal $K$-bundle $\varpi: P \rightarrow D$.

Suppose a group $H$ acts on $P$ from the left, commuting with the right action of $K$, such that the induced action of $H$ on $D$ is biholomorphic. Then $H$ also acts on the holomorphic vector bundle $\mathcal{V} \rightarrow D$, and we form naturally a continuous representation of $H$ on the Fréchet space $\mathcal{O}(D, \mathcal{V})$ consisting of holomorphic sections.

Suppose furthermore that we are given automorphisms of Lie groups $H$ and $K$, and a diffeomorphism of $P$, for which we use the same letter $\sigma$, satisfying the following two conditions:

$$
\sigma(h p k)=\sigma(h) \sigma(p) \sigma(k) \quad(h \in H, k \in K, p \in P) .
$$

The induced action of $\sigma$ on $D(\simeq P / K)$ is anti-holomorphic.

We shall write $P^{\sigma}$ for the set of fixed points by $\sigma$, that is,

$$
P^{\sigma}:=\{p \in P: \sigma(p)=p\}
$$

\section{§2.2. Multiplicity-free theorem - line bundle case}

We start with the simplest case of our multiplicity-free theorem, namely, the case where $\operatorname{dim} V=1$. Here is a statement:

Theorem 1. Suppose that $(\mu, V)$ is one dimensional representation of $K$. Then, $\mathcal{O}(D, \mathcal{V})$ is a multiplicity-free representation of $H$ (see Definition 1.5.3) if there exists $\sigma$ satisfying (2.1), (2.2) and the following two conditions:

$H P^{\sigma} K$ contains an interior point of $P$, $\mu \circ \sigma$ is isomorphic to $\mu^{*}$ as representations of $K$. 
Here, $\mu^{*}$ denotes the contragredient representation of $\mu$.

If we are concerned only with the space $\mathcal{O}(D)$ of holomorphic functions, we may set $K=\{e\}$ and $P=D$. In this case, $(\mu, V)$ is the trivial one-dimensional representation, and the condition (2.4) is automatically satisfied. This case was proved in Faraut and Thomas [12]. A generalization to the line bundle case was given by the author in $[32,36]$. We note that the assumption $(2.3)$ here is formulated in a somewhat different way from $[12,32]$.

Our formulation (2.3) here is intended for a generalization of Theorem 1 to the vector bundle case, which will be presented in Theorem 2 in Section 2.4. The assumption (2.3) will be generalized there by cutting off an "excessive part" of $P^{\sigma}$ (see (2.6) below).

\section{§2.3. Geometry on the base space $D$}

Let us look into a geometric meaning of the condition 2.3 on the base space $D$, which is naturally identified with the quotient space $P / K$. For simplicity, we shall consider the following condition $(2.3)^{\prime}$ instead of $(2.3)$ :

$$
P=H P^{\sigma} K
$$

We recall the setting of Section 2.1: The group $H$ acts holomorphically on a complex manifold $D$, while $\sigma$ acts anti-holomorphically on the same $D$. We note that the $H$-action on $D$ is not necessarily transitive.

Lemma 2.3.1. Assume the condition $(2.3)^{\prime}$ holds. We define a subset of $D$ by $S:=P^{\sigma} K / K$. Then,

$\left.\sigma\right|_{S}=\mathrm{id}$

$\sigma$ preserves each $H$-orbit in $D$.

$3)$

$S$ meets every $H$-orbit in $D$.

Proof. 1) Obvious.

2) Consider the $H$-orbit through $x=p K(p \in P)$ on $D \simeq P / K$. We write $p=h q k\left(h \in H, q \in P^{\sigma}, k \in K\right)$. Then, $\sigma(x)=\sigma(h) q K=\sigma(h) h^{-1} x$. Hence, $\sigma(H x)=H x$.

3) This is a restatement of the condition $H P^{\sigma} K=P\left(\right.$ see $\left.(2.3)^{\prime}\right)$ under the identification $P / K \simeq D$.

We shall return to the above properties $(1) \sim(3)$ in Section 3 (see Definitions 3.1.1 and 3.3.1). In particular, the action of $H$ on $D$ becomes visible in the sense of Definition 3.1.1 owing to Theorem 4. 


\section{§2.4. Multiplicity-free theorem - vector bundle case}

This subsection provides a strengthened version of Theorem 1 by generalizing the setting from line bundles to vector bundles $\mathcal{V} \rightarrow D$. Our goal is to establish a multiplicity-free theorem for $\mathcal{O}(D, \mathcal{V})$.

For this, we need to pose an appropriate assumption on the fiber of the vector bundle $\mathcal{V} \rightarrow D$, because otherwise the multiplicity of irreducible representations of $H$ occurring in $\mathcal{O}(D, \mathcal{V})$ could be arbitrarily large, as one may easily observe the case $H=P=K$ and $D=\{$ one point $\}$ so that $\mathcal{O}(D, \mathcal{V}) \simeq V$ as a representation of $H \simeq K$.

Loosely, our theorem (see Theorem 2) asserts that the representation $\mathcal{O}(D, \mathcal{V})$ is still multiplicity-free if the fiber is relatively "small" compared to a certain subgroup $M$ (see the assumption (2.7)). Here is a rigorous formulation:

Take a subset $B$ of $P^{\sigma}$, and we define

$$
M \equiv M(B):=\{k \in K: b k \in H b \text { for any } b \in B\} .
$$

We pin down a basic property of $M$. We consider the right action of $K$ on the quotient space $H \backslash P$ given by $H p \mapsto H p k$ for $k \in K$. We denote by $K_{H p}$ the isotropy subgroup of $K$ at $H p \in H \backslash P(p \in P)$.

Lemma 2.4.1. 1) $M=\bigcap_{p \in B} K_{H p}$.

2) $M$ is a $\sigma$-stable subgroup.

Proof. The first statement is clear from the definition (2.5). In particular, $M$ is a subgroup of $K$. To see $M$ is $\sigma$-stable, suppose $k \in M$. Then, if $b \in B$, there exists $h \in H$ such that $b k=h b$. Applying $\sigma$ to the both side, we have $b \sigma(k)=\sigma(h) b \in H b$ because $\sigma(b)=b$. This shows $\sigma(k) \in M$. Thus, $M$ is $\sigma$-stable.

Now, we are ready to state our abstract multiplicity-free theorem for vector bundles, which will become a fundamental tool to produce various kinds of multiplicity-free result in later sections.

Theorem 2 (Multiplicity-free theorem for vector bundles [42]). Suppose we are in the setting of Section 2.1. Then, $\mathcal{O}(D, \mathcal{V})$ is a multiplicity-free representation of $H$ (see Definition 1.5.3) if there exist $\sigma$ satisfying (2.1) and (2.2), 
and a subset $B$ of $P^{\sigma}$ with the following four properties:

(2.6) HBK contains an interior point of $P$.

(2.7) The restriction $\left.\mu\right|_{M}$ decomposes as a multiplicity-free sum of irreducible representations of $M$.

We write its irreducible decomposition as $\left.\mu\right|_{M} \simeq \bigoplus_{i} \nu^{(i)}$.

(2.8) $\mu \circ \sigma$ is isomorphic to $\mu^{*}$ as representations of $K$.

(2.9) $\nu^{(i)} \circ \sigma$ is isomorphic to $\left(\nu^{(i)}\right)^{*}$ as representations of $M$ for every $i$.

Remark 2.4.2. The first assumption (2.6) controls the base space $D(\simeq$ $P / K)$, and leads us to the concept of visible actions in Section 3. The second assumption (2.7) controls the fiber. The third and fourth assumptions (2.8) and (2.9) are less important because they are often automatically satisfied.

Remark 2.4.3. The subset $B$ may be regarded as the set of representatives of (generic) $H$-orbits on $D$. The higher the codimension of generic $H$ orbits on $D \simeq P / K$ are, the smaller we can take $B$ to be. Further, the smaller $B$ is, the larger becomes $M$ and the more likely the above assumption (2.7) tends to hold. We shall see this feature in examples such as the classification of multiplicity-free tensor product representations of $\mathfrak{g l}(n, \mathbb{C})$ (see Theorem 27 ).

Remark 2.4.4. The choice of $B$ is sometimes closely related with the structure of Lie groups such as the Cartan decomposition for symmetric pairs or its generalization to certain non-symmetric pairs (see [40]).

Remark 2.4.5. We may regard Theorem 2 as a propagation theorem of multiplicity-free property, i.e., the multiplicity-free representation $\left(\left.\mu\right|_{M}, V\right)$ of a smaller group $M$ "induces" a multiplicity-free representation $\mathcal{O}(D, \mathcal{V})$ of a larger group $H$. Then, the geometric assumption on the $H$-action on a complex manifold $D$ will be investigated in the next section.

\section{$\S 3 . \quad$ Visible Actions on Complex Manifolds}

This section introduces the concept of "visible action" on complex manifolds. Its geometric idea was inspired by the group theoretic assumptions (2.3) or (2.6) for our (abstract) multiplicity-free theorem (see Theorems 1 or 2 in Section 2). Its relation to multiplicity-free representations is examined also in Theorem 5 (Section 3) and Theorem 9 (Section 4).

The definition of visible actions (see Definition 3.1.1) will be followed by a number of examples (see Section 5), which in turn will give rise to various kinds of multiplicity-free representation in later sections (Sections 6, 7 and 8). 


\section{§3.1. Previsible and visible actions on complex manifolds}

Let $D$ be a complex manifold. We recall that a (real) submanifold $S$ is totally real if

$$
T_{x} S \cap J_{x}\left(T_{x} S\right)=\{0\} \quad \text { for any } x \in S,
$$

where $J_{x} \in \operatorname{End}\left(T_{x} D\right)$ stands for the complex structure.

For example, $\mathbb{R}^{n}$ is a totally real submanifold of $\mathbb{C}^{n}$. So is the real Grassmann variety $G r_{p}\left(\mathbb{R}^{n}\right)$ in the complex Grassmann variety $G r_{p}\left(\mathbb{C}^{n}\right)$.

Definition 3.1.1 ([38, Definition 2.3]). Suppose a Lie group $H$ acts holomorphically on a connected complex manifold $D$. We say the action is previsible if there exist a totally real submanifold $S$ in $D$ and a (non-empty) $H$-invariant open subset $D^{\prime}$ of $D$ such that

$$
S \text { meets every } H \text {-orbit in } D^{\prime} \text {. }
$$

A previsible action is said to be visible if

$$
J_{x}\left(T_{x} S\right) \subset T_{x}(H \cdot x)
$$

for all $x \in S$.

Remark 3.1.2. In many interesting cases in later sections, we can take an open set $D^{\prime}$ to be $D$. However, it is important that one can check the condition locally.

An obvious example of visible action is:

Example 3.1.3. A transitive holomorphic action is visible.

This is an extremal case though, previsible actions in general require the existence of considerably large orbits. More precisely, we have:

Proposition 3.1.4. If the action is previsible, then there exists an $\mathrm{H}$ orbit whose dimension is at least half the (real) dimension of $D$.

Proof. The proposition is a direct consequence of Lemma 3.2.1 below. 


\section{§3.2. Infinitesimal characterization for visible actions}

This subsection examines an infinitesimal characterization of visible actions. We start with the following:

Lemma 3.2.1. If the action is previsible, then there exists a non-empty open subset $S^{\prime}$ of $S$ such that

$$
T_{x}(H \cdot x)+T_{x} S=T_{x} D
$$

for all $x \in S^{\prime}$.

Proof. Since the action is previsible, the image of the map

$$
\psi: H \times S \rightarrow D, \quad(h, x) \mapsto h x
$$

contains an open set $D^{\prime}$, and thus, there exists an interior point of the image $\psi(H \times S)$. Since $\psi$ is a $C^{\infty}$-map, we have

$$
\sup _{(h, x) \in H \times S} \operatorname{rank} d \psi_{(h, x)}=\operatorname{dim}_{\mathbb{R}} D .
$$

On the other hand, the rank of $d \psi_{(h, x)}$ is independent of $h \in H$ because

$$
d \psi_{(h, x)}=\left(d L_{h}\right)_{x} \circ d \psi_{(e, x)} \circ d\left(L_{h}^{-1} \times \mathrm{id}\right)_{(h, x)},
$$

where we have used $L_{h}$ to denote the left translation on $D$ and also the one on $H$. Therefore, there exists $x \in S$ such that $\operatorname{rank} d \psi_{(e, x)}=\operatorname{dim}_{\mathbb{R}} D$. As this is an open condition, such $x$ forms an open subset, say $S^{\prime}$, of $S$. Equivalently, (V-2) holds for any $x \in S^{\prime}$ because the left-hand side of $(\mathrm{V}-2)$ is nothing but the image of the differential $d \psi_{(e, x)}: T_{e} H \times T_{x} S \rightarrow T_{x} D$.

We are now led to the following infinitesimal condition:

Definition 3.2.2. $\quad$ Suppose $H$ acts holomorphically on $D$. We say that the action has the property $(\mathbf{V})$ if there exists a totally real submanifold $S$ in $D$ such that $(\mathrm{V}-1)$ and $(\mathrm{V}-2)$ hold for all $x \in S$.

It follows from Lemma 3.2.1 that visibility (see Definition 3.1.1) implies the property $(\mathrm{V})$. In fact, the converse is also true:

Theorem 3. $\quad$ Suppose $H$ acts holomorphically on D. Then, the action is visible if and only if it has the property $(\mathrm{V})$. 
Proof. What remains to prove is that the action becomes previsible, provided the property $(\mathrm{V})$ holds. We retain the notation as in the proof of Lemma 3.2.1. Then, it follows from (V-2) that $d \psi_{(e, x)}: T_{e} H \times T_{x} S \rightarrow T_{x} D$ is surjective for each $x \in S$, whence there exists an open neighborhood $W_{x}$ of $x$ such that $\psi(H \times S) \supset W_{x}$. We set

$$
D^{\prime}:=\bigcup_{h \in H} \bigcup_{x \in S} h W_{x} .
$$

Then, $D^{\prime}$ is an $H$-stable open subset with the property ( $\left.\mathrm{V}-0\right)$. Therefore, the action is previsible, and then becomes visible by the assumption $(\mathrm{V}-1)$. Hence, Theorem has been proved.

\section{§3.3. Anti-holomorphic map}

Thanks to the infinitesimal characterization of visible actions given in Theorem 3 , we can give a convenient sufficient condition for a previsible action to be visible by means of a certain anti-holomorphic diffeomorphism of $D$. To be more precise, we introduce the following:

Definition 3.3.1. $\quad$ A previsible action is strongly visible if there exist an anti-holomorphic diffeomorphism $\sigma$ of an $H$-invariant open subset $D^{\prime}$ and a submanifold $S$ of $D^{\prime}$ (see Definition 3.1.1) such that

$$
\left.\sigma\right|_{S}=\mathrm{id}
$$

$\sigma$ preserves each $H$-orbit in $D^{\prime}$.

Theorem 4. A strongly visible action is visible.

Proof. It follows from Lemma 3.2.1 that there exists a non-empty open subset $S^{\prime}$ of $S$ such that (V-2) holds for all $x \in S^{\prime}$.

We take an arbitrary $X \in T_{x} S$ and decompose $J_{x} X$ according to (V-2):

$$
J_{x} X=Y+Z, \quad\left(Y \in T_{x}(H \cdot x), Z \in T_{x} S\right) .
$$

Since $\sigma$ is an anti-holomorphic diffeomorphism satisfying (S-1), we have

$$
\sigma\left(J_{x} X\right)=-J_{x} X, \quad \sigma Z=Z .
$$

Applying $\sigma$ to (3.2), we have $-J_{x} X=\sigma Y+Z$. Using (3.2) again, we have

$$
2 J_{x} X=Y-\sigma Y \text {. }
$$

Since $\sigma Y$ lies in $T_{x}(H \cdot x)$ by $(\mathrm{S}-2)$, we conclude that $J_{x} X \in T_{x}(H \cdot x)$. Hence, $(\mathrm{V}-1)$ holds for all $x \in S^{\prime}$. Thus, the previsible action is visible by definition. 
Remark 3.3.2. As $\sigma$ is anti-holomorphic, any submanifold $S$ satisfying (S-1) becomes automatically totally real. Indeed, the differential $d \sigma_{x}$ acts on $T_{x} S$ and $J_{x}\left(T_{x} S\right)$ by the scalars 1 and -1 , respectively, and therefore $T_{x} S \cap$ $J_{x}\left(T_{x} S\right)=\{0\}$.

The point of Definition 3.3.1 is its connection to multiplicity-free representations. In fact, the trivial bundle case of Theorem 1 is proved by using Lemma 2.3.1 (2) combined with the following:

Theorem 5 (strongly visible $\Rightarrow$ multiplicity-free). If the $H$-action is strongly visible, then any unitary subrepresentation of $\mathcal{O}(D)$ is multiplicity-free as a representation of $H$.

At this point, the 'slice' $S$ in Definition 3.3.1 is not necessary in proving Theorem 5 . The slice plays a crucial role when we formulate a multiplicity-free theorem in the vector bundle case, as we have seen in Theorem 2. See also Theorem 9 in the next section.

\section{§4. Coisotropic Actions, Polar Actions, and Visible Actions}

This section examines three different type of group actions on manifolds with geometric structure:

1) Coisotropic actions on a symplectic manifold $([15,22])$.

2) Polar actions on a Riemannian manifold ([57, 58]).

3) Visible actions on a complex manifold (Definition 3.1.1).

We are interested in comparing these three notions on a Kähler manifold, which enjoys all three geometric structures, i.e., symplectic, Riemannian and complex structure. For the comparison, we should note that much of the literature on (1) and (2) has made an assumption that both the transformation group $H$ and the manifold $M$ are compact. Accordingly, we shall also assume $H$ and $M$ to be compact throughout this section, although our primary interest is a uniform treatment of both finite and infinite dimensional representations that arise from compact and non-compact geometry.

\section{§4.1. Coisotropic actions on symplectic manifolds}

Let $(M, \omega)$ be a symplectic manifold. A submanifold $N$ is said to be coisotropic if for every $x \in N$

$$
\left(T_{x} N\right)^{\perp \omega} \subset T_{x} N
$$


Here, $\left(T_{x} N\right)^{\perp \omega}:=\left\{u \in T_{x} M: \omega(u, v)=0\right.$ for any $\left.v \in T_{x} N\right\}$. If $2 \operatorname{dim}_{\mathbb{R}} N=$ $\operatorname{dim}_{\mathbb{R}} M$, then $N$ is coisotropic if and only if $N$ is Lagrangian.

Suppose a compact Lie group $H$ acts on $M$ by symplectic automorphisms. The action is called coisotropic (Huckleberry and Wurzbacher [22]) or multiplicity-free (Guillemin and Sternberg [15]) if one and hence all principal orbits $H \cdot x$ are coisotropic with respect to the symplectic form $\omega$, i.e., $T_{x}(H \cdot x)^{\perp \omega} \subset$ $T_{x}(H \cdot x)$.

\section{§4.2. Polar actions on Riemannian manifolds}

Let $M$ be a Riemannian manifold, and $H$ a compact Lie group acting on $M$ by isometries. The action is called polar (e.g. $[57,58]$ ) if there exists a properly embedded submanifold $S$ (called a section) with the following two properties:

(P-0) $S$ meets every $H$-orbit.

(P-1) $\quad T_{x} S \perp T_{x}(H \cdot x)$ for any $x \in S$.

\section{§4.3. Actions on Kähler manifolds}

Suppose $M$ is a Kähler manifold. Then we can naturally equip $M$ with a symplectic structure and a Riemannian structure by taking the imaginary and real part of the Kähler form. Then we can consider coisotropic actions on $M$ as a symplectic manifold, polar actions on $M$ as a Riemannian manifold, and visible actions on $M$ as a complex manifold (see Definition 3.1.1). The aim of this subsection is to compare these three notions. For simplicity, we shall assume that $H$ is a connected and compact Lie group throughout this subsection.

Let $M$ be Kähler. A submanifold $S$ is coisotropic if and only if

$$
J_{x}\left(\left(T_{x} S\right)^{\perp}\right) \subset T_{x} S \text { for all } x \in S,
$$

where $J$ denotes the complex structure on $M$, and $\left(T_{x} S\right)^{\perp}$ the normal space of $S$ at $x$.

We also note that a submanifold $S$ is totally real if and only if

$$
J_{x}\left(T_{x} S\right) \perp T_{x} S \text { for all } x \in S .
$$

Theorem 6 (polar $\Rightarrow$ visible). Let $M$ be a connected Kähler manifold, and $H$ act on $M$ by holomorphic isometries. If the $H$-action is polar with a totally real section $S$, then the $H$-action is visible. 
Remark 4.3.1. A section $S$ for the polar action becomes automatically totally real if $M$ is a compact, irreducible homogeneous Kähler manifold ([57, Proposition 2.1]).

Remark 4.3.2. As we see the proof below, Theorem 6 still holds for a complex manifold with Hermitian metric.

Proof. The $H$-action is obviously previsible. Let us verify the condition (V-1) (see Definition 3.1.1). Suppose $H \cdot x(x \in S)$ is a principal orbit. We define subspaces of $T_{x} M$ by

$$
\begin{aligned}
& A:=T_{x} S, \\
& B:=T_{x}(H \cdot x) .
\end{aligned}
$$

Then, $J A \subset A^{\perp}$ because $A$ is totally real. As the action is polar, we have $A^{\perp}=B$. Thus, $J A \subset A^{\perp}=B$. Hence the $H$-action is visible.

Theorem 7 (visible $\Rightarrow$ coisotropic). $\quad$ Let $M$ be a connected Kähler manifold, and $H$ act on $M$ by holomorphic isometries. If the $H$-action is visible with $\operatorname{dim}_{\mathbb{R}} S=\operatorname{dim}_{\mathbb{C}} M$, then the $H$-action is coisotropic.

Remark 4.3.3. Since $S$ is a totally real submanifold, we have always $\operatorname{dim}_{\mathbb{R}} S \leq \operatorname{dim}_{\mathbb{C}} M$. The above assumption $\operatorname{dim}_{\mathbb{R}} S=\operatorname{dim}_{\mathbb{C}} M$ is quite strong, but is actually often satisfied as we shall see examples in Section 5 as in the case $(S, M)=\left(G r_{p}\left(\mathbb{R}^{n}\right), G r_{p}\left(\mathbb{C}^{n}\right)\right)$.

Proof. We set $A:=T_{x} S$ and $B:=T_{x}(H \cdot x)$ as before. Then $A^{\perp}=J A$ because $S$ is a totally real submanifold with $\operatorname{dim}_{\mathbb{R}} S=\operatorname{dim}_{\mathbb{C}} M$. Furthermore, $J A \subset B$ because the action is visible. Therefore $A^{\perp} \subset B$, and then $B^{\perp} \subset A$. On the other hand, $B^{\perp \omega}=J\left(B^{\perp}\right)$ because $M$ is Kähler. Thus, we have

$$
B^{\perp \omega}=J\left(B^{\perp}\right) \subset J A \subset B .
$$

Hence the action is coisotropic.

The following Theorem is a main ingredient of Podestà and Thorbergsson [57, Theorem 1.1].

Theorem 8 (polar $\Rightarrow$ coisotropic). $\quad$ Let $M$ be a connected Kähler manifold, and $H$ act on $M$ by holomorphic isometries. If the $H$-action is polar with a totally real section $S$, then the $H$-action is coisotropic. 
Proof. The proof is essentially given in [57], but we recall it here for the sake of convenience. Retain the notation of the proof of Theorem 6 .

Since the action is polar, we have $A^{\perp}=B$. As $M$ is Kähler, $B^{\perp \omega}=J\left(B^{\perp}\right)$. Furthermore, $J A \subset A^{\perp}$ because $S$ is totally real. Thus,

$$
B^{\perp \omega}=J\left(B^{\perp}\right)=J A \subset A^{\perp}=B .
$$

Therefore, the $H$-action is coisotropic.

\section{§4.4. Visible actions and multiplicity-free representations}

As a corollary of Theorem 7, we obtain a multiplicity-free theorem for visible action in this setting.

Theorem 9 (visible $\Rightarrow$ multiplicity-free). $\quad$ Let $M$ be a connected Kähler manifold, with a holomorphic, isometric and Poisson action of a connected compact Lie group $H$. Suppose that the $H$-action on $M$ is visible with $\operatorname{dim}_{\mathbb{R}} S=$ $\operatorname{dim}_{\mathbb{C}} M$ (see Definition 3.1.1). Then the representation of $H$ on $\mathcal{O}(M)$ is multiplicity-free.

Proof. Since an isometric Poisson action of a connected compact Lie group $H$ on a compact Kähler manifold $M$ is coisotropic if and only if a Borel subgroup of the complexified Lie group $H_{\mathbb{C}}$ has an open orbit ([22, Equivalence Theorem]), Theorem 9 follows from Theorem 7 .

Remark 4.4.1. In Theorem 5, we have seen that strongly visible actions always give rise to multiplicity-free actions without any assumption of the compactness of the transformation group and the complex manifold. I feel that there is a good room for a generalization of Theorem 9 .

\section{$\S 5 . \quad$ Some Examples of Visible Actions}

In this section, we illustrate visible actions (Definition 3.1.1) by a number of examples. Most of these examples are also strongly visible actions (Definition 3.3.1).

\section{§5.1. Examples of visible actions 1 - abelian case}

We start with the simplest example of visible actions by abelian groups. Consider the (standard) action of a one dimensional toral subgroup $\mathbb{T}:=\{t \in$ $\mathbb{C}:|t|=1\}$ on $\mathbb{C}$ by

$$
\mathbb{T} \times \mathbb{C} \rightarrow \mathbb{C}, \quad(t, z) \mapsto t z .
$$




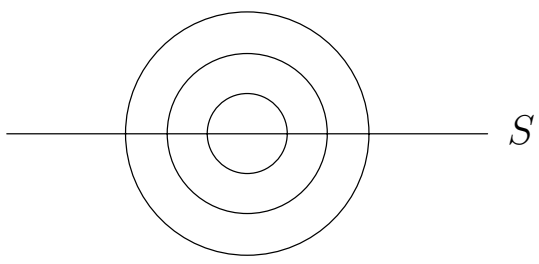

Figure 5.1.1.

Then, as is obvious in Figure 5.1.1, the real line $\mathbb{R}$ meets every $\mathbb{T}$-orbit on $\mathbb{C}$. Furthermore, it is clear that this action is strongly visible (see Definition 3.3.1) by taking $\sigma(z):=\bar{z}$. Thus, Theorem 4 implies that it is also visible. We pin down:

Example 5.1.1. The standard action of $\mathbb{T}$ on $\mathbb{C}$ is (strongly) visible.

Then, a coordinatewise argument leads us immediately to the fact that $\mathbb{R}^{n}$ meets every $\mathbb{T}^{n}$-orbit on $\mathbb{C}^{n}$. Then,

Example 5.1.2. The standard action of $\mathbb{T}^{n}$ on $\mathbb{C}^{n}$ is (strongly) visible.

In turn, its projective version shows that the real projective space $\mathbb{P}^{n-1} \mathbb{R}$ meets every $\mathbb{T}^{n}$-orbit on the projective space $\mathbb{P}^{n-1} \mathbb{C}$, and again by Theorem 4 we have:

Example 5.1.3. The standard action of $\mathbb{T}^{n}$ on $\mathbb{P}^{n-1} \mathbb{C}$ is visible.

Now, we recall the group-theoretic interpretation (triunity given in [38]) for the triple of Lie groups $H \subset G \supset K$, which compares the visibility of the following three actions:

the $H$-action on $G / K$,

the $K$-action on $G / H$,

the diagonal $G$-action on $(G \times G) /(K \times H)$.

Example 5.1.3 treats the $H$-action on the homogeneous space $G / K \simeq \mathbb{P}^{n-1} \mathbb{C}$ if we set $(H, G, K):=\left(\mathbb{T}^{n}, U(n), U(1) \times U(n-1)\right)$. Then, it leads us to two more visible actions of non-abelian groups as follows:

Example 5.1.4. Consider the natural action of $U(n)$ on the complex flag variety $\mathcal{B}\left(\mathbb{C}^{n}\right)(\simeq G / H)$. Then, its restriction to the subgroup $U(1) \times$ $U(n-1)$ is (strongly) visible. A key geometry here is that the real flag variety $\mathcal{B}\left(\mathbb{R}^{n}\right)$ meets every orbit of $U(1) \times U(n-1)$ on $\mathcal{B}\left(\mathbb{C}^{n}\right)$. 
Example 5.1.5. The diagonal action of $U(n)$ on the direct product manifold $\mathbb{P}^{n-1} \mathbb{C} \times \mathcal{B}\left(\mathbb{C}^{n}\right)(\simeq(G \times G) /(K \times H))$ is (strongly) visible. We note that $\mathbb{P}^{n-1} \mathbb{R} \times \mathcal{B}\left(\mathbb{R}^{n}\right)$ meets every diagonal $U(n)$-orbit.

Example 5.1.3 is more or less obvious, yet its equivalences, Examples 5.1.4 and 5.1.5, are the geometry behind non-trivial multiplicity-free representations such as the restriction $U(n) \downarrow U(n-1)$ (see Fact 32) and the tensor product representation $\pi \otimes S^{k}\left(\mathbb{C}^{n}\right)$ (see Example 6.1.1), respectively.

\section{§5.2. Examples of visible actions 2 - group case}

Let $K$ be a compact Lie group, and $K_{\mathbb{C}}$ its complexification. Then $K$ acts on $K_{\mathbb{C}}$ from the left, and also from the right.

Theorem 10 (visible action on complex Lie groups).

1) The left (or right) $K$-action on $K_{\mathbb{C}}$ is previsible.

2) The $(K \times K)$-action on $K_{\mathbb{C}}$ is (strongly) visible.

Proof. 1) Let $\mathfrak{k}$ be the Lie algebra of $K$. Then, $\exp (\sqrt{-1} \mathfrak{k})$ is a totally real submanifold of $K_{\mathbb{C}}$. Therefore, the polar decomposition $K_{\mathbb{C}}=$ $K \exp (\sqrt{-1} \mathfrak{k})$ implies that the left $K$-action on $K_{\mathbb{C}}$ is previsible.

2) Take a normal real form $K_{\mathbb{R}}$ of $K_{\mathbb{C}}$ such that $K \cap K_{\mathbb{R}}$ is a maximal compact subgroup of $K_{\mathbb{C}}$. We write $\sigma$ for the complex conjugation of $K_{\mathbb{C}}$ such that $\left.\sigma\right|_{K_{\mathbb{R}}}=$ id (by taking a covering of $K_{\mathbb{C}}$ if necessary). Take a maximally split abelian subgroup $A_{\mathbb{R}}$ of $K_{\mathbb{R}}$. Then, we have a Cartan decomposition

$$
K_{\mathbb{C}}=K A_{\mathbb{R}} K
$$

because $A_{\mathbb{R}}$ is also a maximally split abelian subgroup of $K_{\mathbb{C}}$. In particular, the totally real submanifold $A_{\mathbb{R}}$ of $K_{\mathbb{C}}$ meets every orbit of $K \times K$. Furthermore, each $(K \times K)$-orbit is preserved under $\sigma$ because $\sigma(K)=K$. Therefore, the action of $K \times K$ on $K_{\mathbb{C}}$ is strongly visible, and then is also visible by Theorem 4 .

Example 5.2.1. $\quad$ For $\left(K, K_{\mathbb{C}}\right)=(U(n), G L(n, \mathbb{C}))$, we may take $K_{\mathbb{R}}=$ $G L(n, \mathbb{R})$ and

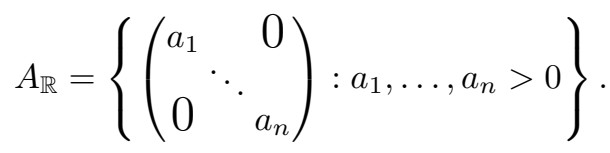


A representation-theoretic counterpart of Theorem 10 (2) is the (wellknown) fact that $L^{2}(K)$ is multiplicity-free as a representation of $K \times K$ (see Fact 28). We note that the left regular representation of $K$ on $L^{2}(K)$ is not multiplicity-free if $K$ is non-abelian because each $\mu \in \widehat{K}$ occurs in $L^{2}(K)$ with multiplicity $\operatorname{dim} \mu$. Therefore, previsibility is not sufficient for representations to be multiplicity-free.

\section{§5.3. Examples of visible actions 3 - complex symmetric case}

Let $G$ be a Lie group, and $\tau$ an involutive automorphism of $G$. Then, $G^{\tau}:=\{g \in G: \tau g=g\}$ is a closed subgroup of $G$. We denote by $\left(G^{\tau}\right)_{0}$ the identity component of $G^{\tau}$. We recall:

Definition 5.3.1. We say $(G, K)$ is a symmetric pair if $K$ is a subgroup of $G$ with $\left(G^{\tau}\right)_{0} \subset K \subset G^{\tau}$. Then the homogeneous space $G / K$ is called a symmetric space. If the group $G$ is furthermore compact [semisimple, reductive, ...], we say $(G, K)$ is a compact [semisimple, reductive, ...] symmetric pair.

Example 5.3.2. For a Lie group $G_{1}$, we set $G:=G_{1} \times G_{1}$ and define $\tau \in \operatorname{Aut}(G)$ by $\tau(x, y):=(y, x)$. Then $G^{\tau}$ is nothing but the diagonal subgroup $\operatorname{diag} G_{1}:=\left\{(x, x): x \in G_{1}\right\}$, and the symmetric space $G / G^{\tau}=$ $\left(G_{1} \times G_{1}\right) / \operatorname{diag} G_{1}$ is naturally identified with the group manifold $G_{1}$ by

$$
G_{1} \times G_{1} / \operatorname{diag} G_{1} \simeq G_{1}, \quad(x, y) \mapsto x y^{-1} .
$$

Here is a generalization of Theorem 10 .

Theorem 11 (visible action on complex symmetric spaces). Suppose that $(G, K)$ is a compact symmetric pair, and that $\left(G_{\mathbb{C}}, K_{\mathbb{C}}\right)$ is its complexification. Then, the $G$-action on the complex symmetric space $G_{\mathbb{C}} / K_{\mathbb{C}}$ is (strongly) visible.

Proof. Let $\mathfrak{g}=\mathfrak{k}+\mathfrak{p}$ be the eigenspace decomposition of the differential of $\tau$ with eigenvalues +1 and -1 . We take a maximally abelian subspace $\mathfrak{a}$ in $\mathfrak{p}$. Let $\sigma$ be a complex conjugation of the Lie algebra $\mathfrak{g}_{\mathbb{C}}$ with respect to the real form $\mathfrak{g}_{\mathbb{R}}:=\mathfrak{k}+\sqrt{-1} \mathfrak{p}$. We lift $\sigma$ to an antiholomorphic involution of the group $G_{\mathbb{C}}$ (by taking a covering group if necessary) and use the same notation $\sigma$. Then, $B:=\exp (\sqrt{-1} \mathfrak{a})$ is a non-compact abelian subgroup and $\left.\sigma\right|_{B}=\mathrm{id}$. Furthermore, we have a (generalized) Cartan decomposition

$$
G_{\mathbb{C}}=G B K_{\mathbb{C}}
$$


Thus, $B \cdot o \simeq B K_{\mathbb{C}} / K_{\mathbb{C}}$ meets every $G$-orbit on $G_{\mathbb{C}} / K_{\mathbb{C}}$. Since $\sigma$ acts antiholomorphically on $G_{\mathbb{C}} / K_{\mathbb{C}}$ and preserves each $G$-orbit, the $G$-action on $G_{\mathbb{C}} / K_{\mathbb{C}}$ is strongly visible, and thus visible by Theorem 4 .

A non-compact version of Theorem 11 will be formulated in Theorem 21 .

\section{§5.4. Examples of visible actions 4 - Hermitian symmetric case}

This subsection studies visible actions on Hermitian symmetric spaces. We start with the Poincaré disk $G / K=S L(2, \mathbb{R}) / S O(2)$ :

Example 5.4.1. Let $G=S L(2, \mathbb{R})$ and we define the following onedimensional subgroups of $G$ :

$$
\begin{aligned}
K & :=\left\{\left(\begin{array}{cc}
\cos \theta-\sin \theta \\
\sin \theta & \cos \theta
\end{array}\right): \theta \in \mathbb{R} / 2 \pi \mathbb{Z}\right\}, \\
H & :=\left\{\left(\begin{array}{cc}
a & 0 \\
0 & a^{-1}
\end{array}\right): a>0\right\}, \\
N & :=\left\{\left(\begin{array}{ll}
1 & x \\
0 & 1
\end{array}\right): x \in \mathbb{R}\right\} .
\end{aligned}
$$

Then, both $(G, K)$ and $(G, H)$ are symmetric pairs, while $N$ is a maximal unipotent subgroup of $G$. Let us consider the left action of $G$ (and its subgroups) on the Hermitian symmetric space $G / K$. Then, all of the actions of the subgroups $K, H$ and $N$ on $G / K$ are (strongly) visible, as one can easily see from the following figures where $G / K$ is realized as the Poincaré disk:

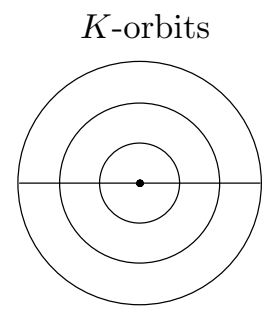

Figure 5.4.1 (a)

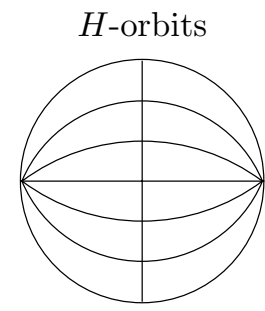

Figure 5.4.1 (b)

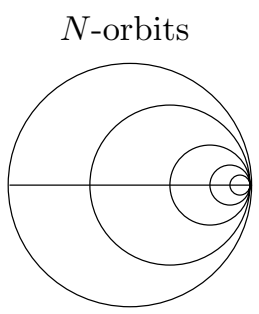

Figure 5.4.1 (c)

The first two cases of Example 5.4.1 are generalized into the following theorems:

Theorem 12 (visible action on Hermitian symmetric spaces, [41]). Let $G / K$ be a Hermitian symmetric space, and $(G, H)$ a symmetric pair. Then, the action of $H$ on $G / K$ is (strongly) visible. 
Theorem 12 includes the following special case:

Corollary 13. Let $X=G / K$ be a Hermitian symmetric space, and $\bar{X}$ another Hermitian symmetric space $G / K$ equipped with the complex conjugate structure of $X$. Then,

1) The diagonal action of $G$ on $X \times X$ is visible.

2) The diagonal action of $G$ on $X \times \bar{X}$ is visible.

The third case of Example 5.4.1 is generalized into the following:

Theorem 14 ([41]). Let $G / K$ be a Hermitian symmetric space without compact factor, and $N$ a maximal unipotent subgroup of $G$. Then, the action of $N$ on $G / K$ is (strongly) visible.

Correspondingly to this geometric result, we shall see multiplicity-free theorems of representations (see Theorems 26, 33 and 34) in both finite and infinite dimensional cases. An interesting geometric feedback includes:

Corollary 15 (sphericity). Let $X$ be a compact Hermitian symmetric space, and $G_{\mathbb{C}}$ the group of biholomorphic transformations on $X$. Then $X$ is an $H_{\mathbb{C}}$-spherical variety (i.e. a Borel subgroup of $H_{\mathbb{C}}$ has an open orbit on $X$ ) for any $H_{\mathbb{C}}$ such that $\left(G_{\mathbb{C}}, H_{\mathbb{C}}\right)$ is a complex symmetric pair.

Proof. The Hermitian symmetric space $X$ is biholomorphic to the generalized flag variety $G_{\mathbb{C}} / P_{\mathbb{C}}$, where $P_{\mathbb{C}}=K_{\mathbb{C}} U$ is a maximal parabolic subgroup of $G_{\mathbb{C}}$ with abelian unipotent radical $U$. If an irreducible rational representation

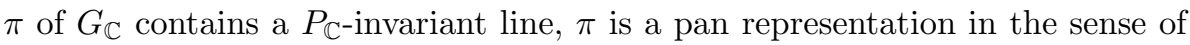
Definition 6.2.1, and its restriction to $H_{\mathbb{C}}$ is multiplicity-free by Theorem 26 in Section 6. Then, it follows from Vinberg and Kimelfeld [70, Corollary 1] that $G_{\mathbb{C}} / P_{\mathbb{C}}$ is $H_{\mathbb{C}-\text { spherical. }}$

Example 5.4.2. We consider the complex Grassmann variety $G r_{p}\left(\mathbb{C}^{n}\right)$ for which $G_{\mathbb{C}}=G L(n, \mathbb{C})$ is the group of biholomorphic transformations. Obviously, the action of $G=U(n)$ is visible (Example 3.1.3). More than this, applying Theorem 12 to the case where

$$
(G, K, H)=(U(n), U(p) \times U(n-p), U(k) \times U(n-k)),
$$

we see that the action of the group $U(k) \times U(n-k)$ on $G r_{p}\left(\mathbb{C}^{n}\right)$ is (strongly) visible for any $p$ and $k$ because $(G, H)$ is a symmetric pair and $G / K \simeq G r_{p}\left(\mathbb{C}^{n}\right)$. 
Furthermore, it follows from Corollary 15 that $G r_{p}\left(\mathbb{C}^{n}\right)$ is a spherical variety of the group $H_{\mathbb{C}}=G L(k, \mathbb{C}) \times G L(n-k, \mathbb{C})$ for any $p$ and $k$. This fact played a key role in determining explicitly the image and the kernel of the Radon-Penrose transform for some non-compact complex homogeneous manifolds ([63, 64]).

\section{§5.5. Examples of visible actions 5 - non-symmetric case}

This subsection gives a refinement of Example 5.4.2.

Let $n=n_{1}+n_{2}+\cdots+n_{k}$ be a partition of $n$, and $L:=U\left(n_{1}\right) \times U\left(n_{2}\right) \times$ $\cdots \times U\left(n_{k}\right)$ be the natural subgroup of $G:=U(n)$. We note that $(G, L)$ is a non-symmetric pair if $k \geq 3$. The homogeneous space $G / L$ is naturally identified with the complex (generalized) flag variety $\mathcal{B}_{n_{1}, n_{2}, \ldots, n_{k}}\left(\mathbb{C}^{n}\right)$ consisting of a sequence of complex vector subspaces $l_{0}=\{0\} \subset l_{1} \subset l_{2} \subset \cdots \subset l_{k} \subset \mathbb{C}^{n}$ such that $\operatorname{dim} l_{j}=\operatorname{dim} l_{j-1}+n_{j}(1 \leq j \leq k)$. We note that $\mathcal{B}_{p, n-p}\left(\mathbb{C}^{n}\right)=$ $G r_{p}\left(\mathbb{C}^{n}\right)$ and that $\mathcal{B}_{1,2, \ldots, n}\left(\mathbb{C}^{n}\right)$ is the (full) flag variety $\mathcal{B}\left(\mathbb{C}^{n}\right)$.

Let us consider the natural action of $U(p) \times U(q)(p+q=n)$ on the flag variety $\mathcal{B}_{n_{1}, n_{2}, \ldots, n_{k}}\left(\mathbb{C}^{n}\right)$, and as its dual we shall also consider the natural action of $L$ on the Grassmann variety $G r_{p}\left(\mathbb{C}^{n}\right)$.

Then the following theorem holds.

Theorem 16 (actions on the generalized flag variety, [40]). Suppose $n_{1}+n_{2}+\cdots+n_{k}=p+q=n$. Then the following four conditions on $p, q, k, n_{1}, \ldots, n_{k}$ are equivalent:

i) The real Grassmann variety $G r_{p}\left(\mathbb{R}^{n}\right)$ meets every orbit of the group $U\left(n_{1}\right) \times$ $U\left(n_{2}\right) \times \cdots \times U\left(n_{k}\right)$ on the complex Grassmann variety $G r_{p}\left(\mathbb{C}^{n}\right)$.

ii) The real (partial) flag variety $\mathcal{B}_{n_{1}, n_{2}, \ldots, n_{k}}\left(\mathbb{R}^{n}\right)$ meets every orbit of the group $U(p) \times U(q)$ on the complex flag variety $\mathcal{B}_{n_{1}, n_{2}, \ldots, n_{k}}\left(\mathbb{C}^{n}\right)$.

iii) One of (a), (b), (c) or (d) is satisfied:
a) $\min (p, q)=1$ (and $n_{1}, \ldots, n_{k}$ are arbitrary).
b) $\min (p, q)=2$ and $k \leq 3$.
c) $\min (p, q) \geq 3$ and $k \leq 2$.
d) $\min (p, q) \geq 3, k=3$ and $\min \left(n_{1}, n_{2}, n_{3}\right)=1$.

iv) (Spherical variety) A Borel subgroup of $G L\left(n_{1}, \mathbb{C}\right) \times G L\left(n_{2}, \mathbb{C}\right) \times \cdots \times$ $G L\left(n_{k}, \mathbb{C}\right)$ has an open orbit on $G r_{p}\left(\mathbb{C}^{n}\right)$.

The following Corollary is straightforward by taking the natural complex conjugation $\sigma$ of $G r_{p}\left(\mathbb{C}^{n}\right), \mathcal{B}_{n_{1}, n_{2}, \ldots, n_{k}}\left(\mathbb{C}^{n}\right)$, and $G r_{p}\left(\mathbb{C}^{n}\right) \times \mathcal{B}_{n_{1}, n_{2}, \ldots, n_{k}}\left(\mathbb{C}^{n}\right)$ respectively. 
Corollary 17 (visible actions on the generalized flag variety). Retain the setting of Theorem 16. If the condition (iii) is satisfied, then the followings hold.

1) The action of $U\left(n_{1}\right) \times \cdots \times U\left(n_{k}\right)$ on $G r_{p}\left(\mathbb{C}^{n}\right)$ is (strongly) visible.

2) The action of $U(p) \times U(q)$ on $\mathcal{B}_{n_{1}, n_{2}, \ldots, n_{k}}\left(\mathbb{C}^{n}\right)$ is (strongly) visible.

3) The diagonal action of $U(n)$ on $G r_{p}\left(\mathbb{C}^{n}\right) \times \mathcal{B}_{n_{1}, n_{2}, \ldots, n_{k}}\left(\mathbb{C}^{n}\right)$ is (strongly) visible.

We note that we have already examined two special cases of (iii), namely, $k=2$ in Example 5.4.2 and $p=1, k=n, n_{1}=\cdots=n_{k}=1$ in Example 5.1.3.

\section{§5.6. Examples of visible actions 6 - linear multiplicity-free space}

This subsection treats linear actions on vector spaces $V$ and the representations on the space $\operatorname{Pol}(V)$ of polynomials on $V$. Let $H$ be a subgroup of $G L_{\mathbb{C}}(V) . V$ is called a multiplicity-free space of $H$ if the natural representation of $H$ on $\operatorname{Pol}(V)$ is multiplicity-free. We shall present some few examples of visible actions of $H$ on $V$ and multiplicity-free spaces $V$ (see Example 5.6.1 and Theorem 19).

We start with the linear action of $U(p) \times U(q)$ on $M(p, q ; \mathbb{C})$ given by

$$
X \mapsto A X B^{-1} \quad(A \in U(p), B \in U(q)) .
$$

The following example is basic. Surprisingly, the geometry will also lead to all spherical nilpotent orbits of $G L(n, \mathbb{C})$ via the momentum map. We note that spherical nilpotent orbits were classified by Panyushev [56] by a different method (see Theorem 20).

\section{Example 5.6.1.}

1) The action of $U(p) \times U(q)$ on $M(p, q ; \mathbb{C})$ is visible.

2) $\operatorname{Pol}(M(p, q ; \mathbb{C}))$ is multiplicity-free as a $G L(p, \mathbb{C}) \times G L(q, \mathbb{C})$ module.

We shall observe that this example has three descendants, i.e., the isotropy action on the tangent space of a Hermitian symmetric space (Theorem 18), Kac's example for multiplicity-free space (Theorem 19), and Panyushev's example of spherical nilpotent orbits (Theorem 20). 
Proof. 1) Every orbit of $U(p) \times U(q)$ on $M(p, q ; \mathbb{C})$ meets the subspace $B$ of dimension $\min (p, q)$ in $M(p, q ; \mathbb{R})$ given by

$$
\left\{\left(\begin{array}{ccc}
a_{1} & & \\
& \ddots & \\
& & a_{p}
\end{array}\right): a_{1}, \ldots, a_{p} \in \mathbb{R}\right\} \quad(p \leq q),
$$

or

$$
\left\{\left(\begin{array}{ccc}
a_{1} & & \\
& \ddots & \\
& & a_{q} \\
& O
\end{array}\right): a_{1}, \ldots, a_{q} \in \mathbb{R}\right\} \quad(p \geq q)
$$

Furthermore, it turns out that this action is strongly visible by the antiholomorphic map $\sigma$ given by $\sigma(X):=\bar{X}(X \in M(p, q ; \mathbb{C}))$, and therefore is visible by Theorem 4 .

2) This statement follows from Theorem 5 .

Remark 5.6.2. The explicit decomposition formula of $\operatorname{Pol}(M(p, q ; \mathbb{C}))$ is also well-known. With notation as in Section 6.1 , here is the formula:

$$
\operatorname{Pol}(M(p, q ; \mathbb{C})) \simeq\left\{\begin{array}{cc}
\bigoplus_{\lambda_{1} \geq \cdots \geq \lambda_{p} \geq 0} \pi_{\left(-\lambda_{p}, \ldots,-\lambda_{1}\right)} \otimes \pi_{\left(\lambda_{1}, \ldots, \lambda_{p}, 0, \ldots, 0\right)} & (p \leq q), \\
\bigoplus_{\lambda_{1} \geq \cdots \geq \lambda_{q} \geq 0} \pi_{\left(0, \ldots, 0,-\lambda_{q}, \ldots,-\lambda_{1}\right)} \otimes \pi_{\left(\lambda_{1}, \ldots, \lambda_{q}\right)} & (p \geq q) .
\end{array}\right.
$$

This formula is referred as the $\left(G L_{p}, G L_{q}\right)$-duality from Howe's viewpoint of dual pair correspondence $([19,20])$, and also as a special case of the $K$-type formula of a holomorphic discrete series representation of the non-compact Lie group $U(p, q)$. See Remark 5.6.3 after Theorem 18 .

Suppose $G / K$ is a Hermitian symmetric space of non-compact type. We write $\mathfrak{g}=\mathfrak{k}+\mathfrak{p}$ for the Cartan decomposition of the Lie algebra $\mathfrak{g}$, and identify $\mathfrak{p}$ with the holomorphic tangent space $T_{o}(G / K)$ at $o=e K$. Take a maximal abelian subspace $\mathfrak{a}$ in $\mathfrak{p}$, and we set $M=\left\{k \in K:\left.\operatorname{Ad}(k)\right|_{\mathfrak{a}}=\mathrm{id}\right\}$.

The isotropy representation of $K$ on $T_{o}(G / K)$ is holomorphic, and is identified with the adjoint representation of $K$ on $\mathfrak{p}$. The action (5.2) is such an example where $(G, K)=(U(p, q), U(p) \times U(q))$. Then, Example 5.6.1 is a special case of the following:

Theorem 18 (tangent action for Hermitian symmetric space). Retain the above setting where $G / K$ is a Hermitian symmetric space. 
1) The adjoint action of $K$ on $\mathfrak{p}$ is (strongly) visible.

2) (Johnson [27], see also Schmid [62]) $\operatorname{Pol}(\mathfrak{p})$ is multiplicity-free as a Kmodule.

The statement (2) can be strengthened as:

3) Let $\mu$ be a $K$-module such that the restriction $\left.\mu\right|_{M}$ is multiplicity-free as an $M$-module. Then, $\operatorname{Pol}(\mathfrak{p}) \otimes \mu$ is multiplicity-free as a $K$-module.

Sketch of proof. 1) Realize $G / K$ as a bounded symmetric domain in $\mathfrak{p}$. Then, the statement follows from Theorem 12 (in the case $H=K$ ) because the (strong) visibility is a local condition.

2) Follows from (1) and Theorem 5.

3) This statement follows from Theorem 2. Here, the key point is that $\mathfrak{a}$ meets every $K$-orbit in $\mathfrak{p}$, and the group $M$ in Theorem 2 is nothing but our $M$ in Theorem 18.

Remark 5.6.3. More than the abstract multiplicity-free property, the explicit decomposition formula of $\operatorname{Pol}(\mathfrak{p})$ is also known (see Johnson [27], Schmid [62]). We have given its generalization to infinite dimensional representations in [32], which we shall review in Theorem 39 in Section 8.5.

Let us consider another generalization of Example 5.6.1. We extend (5.2) to the action of $U(p) \times U(q)$ on $M(p, q+1 ; \mathbb{C}) \simeq M(p, q ; \mathbb{C}) \oplus \mathbb{C}^{p}$ in the following two ways: for $(A, B) \in U(p) \times U(q)$,

$$
\begin{array}{ll}
M(p, q+1 ; \mathbb{C}) \rightarrow M(p, q+1 ; \mathbb{C}), & (X, Y) \mapsto\left(A X B^{-1}, A Y\right), \\
M(p, q+1 ; \mathbb{C}) \rightarrow M(p, q+1 ; \mathbb{C}), & (X, Y) \mapsto\left(A X B^{-1},{ }^{t} A^{-1} Y\right) .
\end{array}
$$

Then our geometric approach gives a new proof that $M(p, q+1 ; \mathbb{C})$ is a multiplicity-free space of $U(p) \times U(q)$ :

Theorem 19 (linear multiplicity-free space). In both cases, the following statements hold.

1) ([38]) The action of $U(p) \times U(q)$ on $M(p, q+1 ; \mathbb{C})$ is (strongly) visible.

2) $($ Kac $[29]) \operatorname{Pol}(M(p, q+1 ; \mathbb{C}))$ is multiplicity-free as a $G L(p, \mathbb{C}) \times G L(q, \mathbb{C})$ module.

Proof. The proof is similar to that of Example 5.6.1. A key point is that $B \oplus \mathbb{R}^{p} \simeq \mathbb{R}^{\min (p, q)} \oplus \mathbb{R}^{p}$ meets every orbit of $U(p) \times U(q)$ on $M(p, q+1 ; \mathbb{C})$ (see [38, Proposition 2.8]). The second statement follows from Theorem 5. 
Remark 5.6.4. As is well-known, and also as we have given a new approach in Theorem 18, $\mathfrak{p}$ is a multiplicity-free space of $K$ if $G / K$ is a Hermitian symmetric space. In addition to the examples that arise from symmetric spaces, there are some few multiplicity-free spaces. For example, $M(p+i, q+j)$ is a multiplicity-free space of (the natural linear action of) $U(p) \times U(q)$ if and only if $(i, j)=(0,0),(0,1)$ or $(1,0)$. The case $(i, j)=(0,0)$ arises from the symmetric space $U(p+q) / U(p) \times U(q)$, while $(i, j)=(0,1)$ or $(1,0)$ does not (i.e. the nonsymmetric case). Victor Kac [29] classified irreducible (linear) multiplicity-free spaces building on earlier work including a classification of irreducible prehomogeneous vector spaces due to Sato-Kimura [61]. The main machinery of this classification is the open Borel orbit criterion.

\section{§5.7. Examples of visible actions 7 - spherical nilpotent varieties}

Let $\mathcal{N}_{\left(2^{p} 1^{n-2 p}\right)}$ be the set of nilpotent matrices of Jordan type $\left(2^{p} 1^{n-2 p}\right)$ in $M(n, \mathbb{C})$. Then, $\mathcal{N}_{\left(2^{p} 1^{n-2 p}\right)}$ is the single orbit of $G L(n, \mathbb{C})$ that goes through

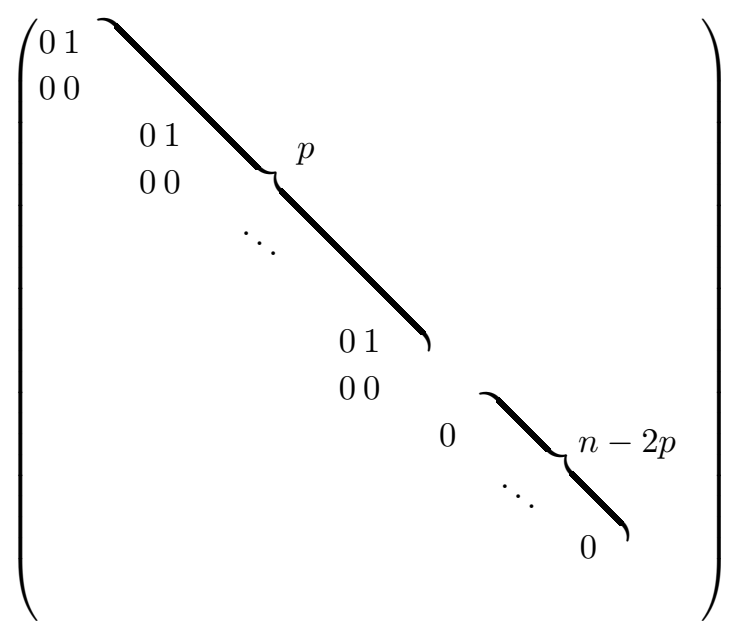

by means of the adjoint representation on $\mathfrak{g l}(n, \mathbb{C})$. In particular, it carries a complex manifold structure.

Theorem 20 (spherical nilpotent orbits). Let $0 \leq 2 p \leq n$.

1) The action of $U(n)$ on the nilpotent orbit $\mathcal{N}_{\left(2^{p} 1^{n-2 p}\right)}$ is (strongly) visible.

2) (Panyushev) $\mathcal{O}\left(\mathcal{N}_{\left(2^{p} 1^{n-2 p}\right)}\right)$ is multiplicity-free. 
Panyushev [56] classified all nilpotent orbits $\mathcal{N}$ such that $\mathcal{O}(\mathcal{N})$ is multiplicity-free. For the $G L_{n}$ case, the above nilpotent orbits $\mathcal{N}_{\left(2^{p} 1^{n-2 p}\right)}(0 \leq 2 p \leq n)$ exhaust all such $\mathcal{N}$.

Our proof (see below) for the multiplicity-free statement (see (2)) is again built on the abstract multiplicity-free theorem in Section 2 combined with visible actions on nilpotent orbits (see (1)), and is different from [56].

A key idea is the observation that the visibility of $U(n)$ action on nilpotent orbits is inherited from the visibility of $U(p) \times U(q)$ action on $M(p, q ; \mathbb{C})$ via the momentum map.

Proof. Let $q=n-p$. We denote by $H$ the natural subgroup $U(p) \times U(q)$ in $G:=U(n)$.

We define an embedding $\iota: M(p, q ; \mathbb{C}) \rightarrow \mathfrak{g l}(n, \mathbb{C})(n=p+q)$ by $X \mapsto$ $\left(\begin{array}{ll}O & X \\ O & O\end{array}\right)$. Then, the action of $H$ on $M(p, q ; \mathbb{C})$ by means of the adjoint action is identified with (5.2).

Now, we define the following map:

$$
\varphi: G \times_{H} M(p, q ; \mathbb{C}) \rightarrow \mathfrak{g l}(n, \mathbb{C}), \quad(g, X) \mapsto g X g^{-1} .
$$

Via the following identifications:

$$
\begin{aligned}
G r_{p}\left(\mathbb{C}^{n}\right) & \simeq G / H, \\
T^{*} G r_{p}\left(\mathbb{C}^{n}\right) & \simeq G \times_{H} M(p, q ; \mathbb{C}), \\
\mathfrak{g l}(n, \mathbb{C}) & \simeq \mathfrak{g l}(n, \mathbb{C})^{*},
\end{aligned}
$$

the map $\varphi$ is essentially the momentum map of the Hamiltonian action of $U(n)$ on $G r_{p}\left(\mathbb{C}^{n}\right)$.

Furthermore, it turns out that the image of $\varphi$ coincides with the closure of $\mathcal{N}_{\left(2^{p} 1^{n-2 p}\right)}$, namely,

$$
\overline{\mathcal{N}_{\left(2^{p} 1^{n-2 p}\right)}}=\left\{X \in \mathfrak{g l}(n, \mathbb{C}): \operatorname{rank} X \leq p, X^{2}=0\right\} .
$$

Let $B \simeq \mathbb{R}^{\min (p, q)}\left(=\mathbb{R}^{q}\right)$ be the real subspace of $M(p, q ; \mathbb{C})$ as in the proof of Example 5.6.1. Then, the proof of Example 5.6.1 asserts

$$
H \cdot B=M(p, q ; \mathbb{C}) .
$$

Via the momentum map $\varphi$, this in turn implies

$$
G \cdot \iota(B)=\overline{\mathcal{N}_{\left(2^{p} 1^{n-2 p}\right)}} \quad \text { and } \quad G \cdot \iota\left(B^{\prime}\right)=\mathcal{N}_{\left(2^{p} 1^{n-2 p}\right)},
$$


where we set an open dense set $B^{\prime}:=\left(\mathbb{R}^{\times}\right)^{q}$ in $B$. Hence, the $G$-action on $\mathcal{N}_{\left(2^{p} 1^{n-2 p}\right)}$ is previsible. Furthermore, if we set $\sigma(X):=\bar{X}\left(X \in \mathcal{N}_{\left(2^{p} 1^{n-2 p}\right)}\right)$, we see easily that the $G$-action on $\mathcal{N}_{\left(2^{p} 1^{n-2 p}\right)}$ is strongly visible (Definition 3.3.1). Therefore the $G$-action is visible by Theorem 4 and $\mathcal{O}\left(\mathcal{N}_{\left(2^{p} 1^{n-2 p}\right)}\right)$ is multiplicity-free by Theorem 5 .

\section{§5.8. Examples of visible actions $8-$ Stein extension of a Riemannian symmetric space}

In Subsection 5.3, we have proved the visibility of the action of a compact Lie group $G$ on the complexification of a compact symmetric space $G / K$ (see Theorem 11). In this subsection, we shall consider its non-compact version.

Let $G$ be a semisimple Lie group without compact factor, and $K$ a maximal compact subgroup of $G$. We write $\mathfrak{g}=\mathfrak{k}+\mathfrak{p}$ for the Cartan decomposition of the Lie algebra $\mathfrak{g}$, and take a maximal abelian subspace $\mathfrak{a}$ of $\mathfrak{p}$.

For simplicity, we assume that $G$ and $K$ are contained in complexified Lie groups $G_{\mathbb{C}}$ and $K_{\mathbb{C}}$. The crown $D$ of the Riemannian symmetric space $G / K$ is a $G$-invariant Stein domain in the complex manifold $G_{\mathbb{C}} / K_{\mathbb{C}}$, defined by

$$
D:=G \exp \sqrt{-1} \mathfrak{a}_{\frac{\pi}{2}} \cdot o,
$$

where $o=e K_{\mathbb{C}}$ and

$$
\mathfrak{a}_{\frac{\pi}{2}}:=\left\{H \in \mathfrak{a}:|\operatorname{Spec}(\operatorname{ad} H)|<\frac{\pi}{2}\right\} .
$$

The domain $D$ is also referred as the Akhiezer-Gindikin domain.

For any one-dimensional representation $\chi$ of $K$, we can extend $\chi$ to a holomorphic representation of $K_{\mathbb{C}}$, and form a $G$-equivariant holomorphic line bundle $\mathcal{L}_{\chi}$ on $D$.

Theorem 21 (Riemannian symmetric space). Let $G / K$ be a Riemannian symmetric space of non-compact type.

1) The action of $G$ on the crown $D$ of $G / K$ is (strongly) visible.

2) For any one dimensional representation $\chi$ of $K, \mathcal{O}\left(D, \mathcal{L}_{\chi}\right)$ is multiplicityfree. In particular, $\mathcal{O}(D)$ is multiplicity-free.

The following Fact is a non-compact version of É. Cartan's theorem ([7], see Fact 29). Various proofs are known, including a proof due to I. M. Gelfand ([13]) based on the commutativity of the Hecke algebra $L^{1}(K \backslash G / K)$ of $K$ biinvariant integrable functions with respect to convolution. Harish-Chandra 
and Helgason's explicit Plancherel formula (e.g. [18]) also gives another proof of it. Yet, Theorem 21 provides a new proof of this multiplicity-free property:

Corollary 22. The regular representation $L^{2}(G / K)$ is multiplicity-free.

Proof. There is an injective intertwining operator $L^{2}(G / K) \rightarrow \mathcal{O}(D)$ (see Krötz and Stanton [47] for the construction of such intertwining operator by using the heat kernel). Thus, $L^{2}(G / K)$ is multiplicity-free by Theorem 21 (2).

It is well-known that the regular representation $L^{2}(G / K)$ is decomposed into the direct integral of spherical principal series representations with multiplicity free. There is no discrete spectrum.

\section{§6. Multiplicity-Free Representations — Finite Dimensional Examples}

The aim of this section is to illustrate by finite dimensional examples the idea as to how the abstract theorem (Theorem 2) gives rise to various kinds of multiplicity-free result. The underlying geometry such as visible actions on flag varieties will be also discussed.

\section{§6.1. Multiplicity-free tensor product representations}

Throughout this section, $\mathfrak{g}_{\mathbb{C}}$ will denote a complex reductive Lie algebra. We take a Cartan subalgebra $\mathfrak{t}_{\mathbb{C}}$, and fix a positive system $\Delta^{+}\left(\mathfrak{g}_{\mathbb{C}}, \mathfrak{t}_{\mathbb{C}}\right)$. For a dominant integral weight $\lambda \in \mathfrak{t}_{\mathbb{C}}^{*}$, we write $\pi_{\lambda}$ for the irreducible finite dimensional representation of $\mathfrak{g}_{\mathbb{C}}$ with highest weight $\lambda$.

By using an invariant non-degenerate bilinear form on $\mathfrak{g}_{\mathbb{C}}$ (e.g., the Killing form if $\mathfrak{g}_{\mathbb{C}}$ is semisimple), we identify $\mathfrak{g}_{\mathbb{C}}^{*}$ with $\mathfrak{g}_{\mathbb{C}}$, and also $\mathfrak{t}_{\mathbb{C}}^{*}$ with $\mathfrak{t}_{\mathbb{C}}$. For $\lambda \in \mathfrak{t}_{\mathbb{C}}^{*}$, we write $X_{\lambda} \in \mathfrak{t}_{\mathbb{C}}\left(\subset \mathfrak{g}_{\mathbb{C}}\right)$ for the corresponding element, and define a Levi subalgebra $\left(\mathfrak{g}_{\mathbb{C}}\right)_{\lambda}$ of $\mathfrak{g}_{\mathbb{C}}$ by

$$
\begin{aligned}
\left(\mathfrak{g}_{\mathbb{C}}\right)_{\lambda} & :=\left\{Y \in \mathfrak{g}_{\mathbb{C}}:\left[X_{\lambda}, Y\right]=0\right\} \\
& =\left\{Y \in \mathfrak{g}_{\mathbb{C}}: \lambda([Y, Z])=0 \text { for any } Z \in \mathfrak{g}_{\mathbb{C}}\right\} .
\end{aligned}
$$

Theorem 23 ([38, Theorem 3.5]). The tensor product representation $\pi_{\lambda} \otimes \pi_{\nu}$ decomposes as a multiplicity-free sum of irreducible representations of $\mathfrak{g}_{\mathbb{C}}$ if $\pi_{\nu}$ is multiplicity-free when restricted to $\left(\mathfrak{g}_{\mathbb{C}}\right)_{\lambda}$. 
Sketch of proof. For simplicity, assume $\mathfrak{g}_{\mathbb{C}}$ is semisimple. Let $G$ be a simply connected, compact Lie group such that $\mathfrak{g}_{\mathbb{C}} \simeq \operatorname{Lie}(G) \otimes_{\mathbb{R}} \mathbb{C}$, and $L$ the isotropy subgroup of $G$ at $X_{\lambda}$ by means of the adjoint representation. (We note that $\left(\mathfrak{g}_{\mathbb{C}}\right)_{\lambda} \simeq \operatorname{Lie}(L) \otimes_{\mathbb{R}} \mathbb{C}$.) Then, Theorem 23 follows from Theorem 2 by applying $(P, H, K, \mu, B)=\left(G \times G, \operatorname{diag}(G), L \times G, \mathbb{C}_{\lambda} \otimes \pi_{\nu},\{e\}\right)$. See [38] for details.

The assumption of Theorem 23 is automatically fulfilled for any $\lambda$ if $\pi_{\nu}$ is weight multiplicity-free, i.e., if $\pi_{\nu}$ decomposes with multiplicity free when restricted to a Cartan subalgebra (see [20, Theorem 4.6.3] for the classification of weight multiplicity-free representations). In this special case, Theorem 23 reduces to the following well-known fact:

Fact 24. If $F$ is weight multiplicity-free, then the tensor product representation $\pi \otimes F$ is multiplicity-free for any irreducible representation $\pi$ of $\mathfrak{g}_{\mathbb{C}}$.

Example 6.1.1. The $k$ th symmetric tensor representation $S^{k}\left(\mathbb{C}^{n}\right)$ is a weight multiplicity-free representation of $\mathfrak{g}_{\mathbb{C}}=\mathfrak{g l}(n, \mathbb{C})$ for any $k=0,1,2, \ldots$. (As its highest weight is given by $(k, 0, \ldots, 0), S^{k}\left(\mathbb{C}^{n}\right)$ is expressed as $\pi_{(k, 0, \ldots, 0)}$ in our notation.) The tensor product representation $\pi_{\lambda} \otimes S^{k}\left(\mathbb{C}^{n}\right)$ decomposes into a multiplicity-free sum of irreducible representations of $\mathfrak{g}_{\mathbb{C}}$ for any dominant integral weight $\lambda=\left(\lambda_{1}, \ldots, \lambda_{n}\right)$ and for any $k$. The decomposition formula is given by

$$
\pi_{\lambda} \otimes \pi_{(k, 0, \ldots, 0)} \simeq \underset{\substack { \mu_{1} \geq \lambda_{1} \geq \cdots \geq \mu_{n} \geq \lambda_{n} \\
\begin{subarray}{c}{n \\
\sum_{i=1}^{n}\left(\mu_{i}-\lambda_{i}\right)=k{ \mu _ { 1 } \geq \lambda _ { 1 } \geq \cdots \geq \mu _ { n } \geq \lambda _ { n } \\
\begin{subarray} { c } { n \\
\sum _ { i = 1 } ^ { n } ( \mu _ { i } - \lambda _ { i } ) = k } }\end{subarray}}{\bigoplus} \pi_{\left(\mu_{1}, \ldots, \mu_{n}\right)} .
$$

This formula is known as Pieri's rule (the Clebsch-Gordan formula if $n=2)$.

\section{§6.2. Parabolic subalgebras with abelian nilradicals}

Let $\pi_{\lambda}$ be an irreducible finite dimensional representation of a complex reductive Lie algebra $\mathfrak{g}_{\mathbb{C}}$. Retain the notation as in Subsection 6.1.

Definition 6.2.1. We say $\pi_{\lambda}$ is a pan representation if $\left(\mathfrak{g}_{\mathbb{C}},\left(\mathfrak{g}_{\mathbb{C}}\right)_{\lambda}\right)$ is a symmetric pair (see (6.1)).

Here, "pan" stands for a parabolic subalgebra with abelian nilradical.

We note that the above condition on $\lambda$ is equivalent to that the nilradical of $\mathfrak{p}(\lambda)$ is abelian, where $\mathfrak{p}(\lambda)$ is the parabolic subalgebra of $\mathfrak{g}_{\mathbb{C}}$ defined as the 
sum of eigenspaces of $\sqrt{-1} \operatorname{ad}\left(X_{\lambda}\right)$ with non-negative eigenvalues. We also note that the above condition on $\lambda$ is clearly equivalent to the following condition: there exists a Levi subalgebra $\mathfrak{l}_{\mathbb{C}}$ containing $\mathfrak{t}_{\mathbb{C}}$ such that

1) $\left.\lambda\right|_{\mathfrak{t}_{\mathbb{C}} \cap\left[\mathfrak{l}_{\mathbb{C}}, \mathfrak{l}_{\mathbb{C}}\right]} \equiv 0$

2) $\left(\mathfrak{g}_{\mathbb{C}}, \mathfrak{l}_{\mathbb{C}}\right)$ is a symmetric pair.

Let us rewrite the above condition more explicitly, assuming that $\mathfrak{g}_{\mathbb{C}}$ is simple. We fix a positive system $\Delta^{+}\left(\mathfrak{g}_{\mathbb{C}}, \mathfrak{t}_{\mathbb{C}}\right)$ and label the simple roots $\alpha_{1}, \ldots, \alpha_{n}$ in the Dynkin diagram as follows:

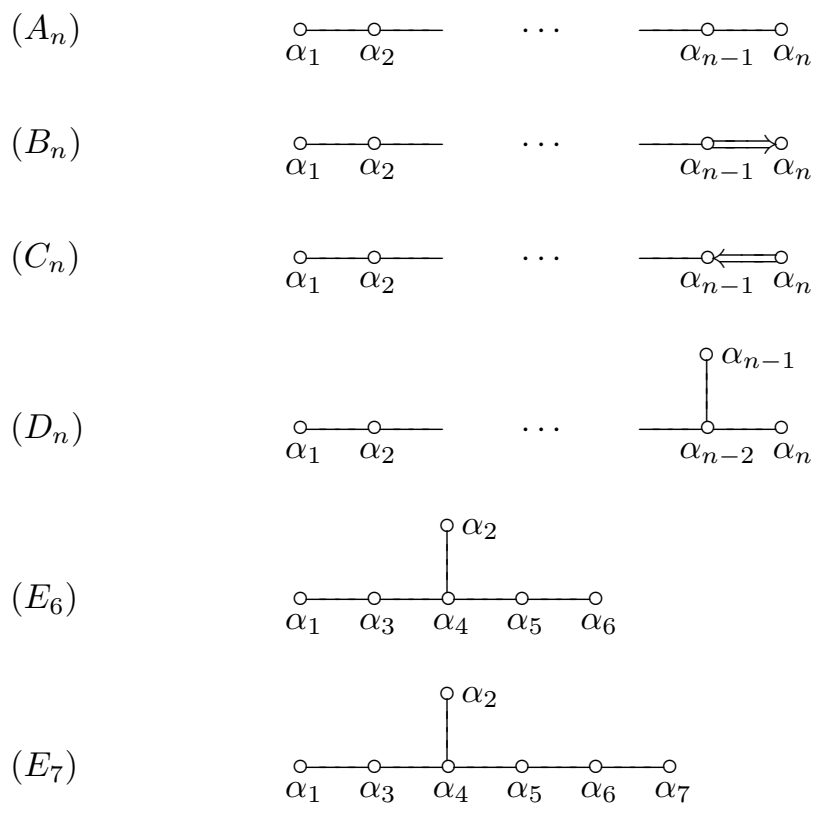

We denote by $\omega_{1}, \ldots, \omega_{n}$ the fundamental dominant weights, that is, $\omega_{j}$ satisfies

$$
\frac{2\left(\omega_{i}, \alpha_{j}\right)}{\left(\alpha_{j}, \alpha_{j}\right)}=\delta_{i j}
$$

Then, we have the following lemma:

Lemma 6.2.2. $\quad \pi_{\lambda}$ is a pan representation if and only if $\lambda$ is of the form $\lambda=k \omega_{i}(k \in \mathbb{N})$, where $i$ is in the following list: 


$$
\begin{array}{ll}
i=1,2, \ldots, n & \text { for }\left(A_{n}\right), \\
i=1 & \text { for }\left(B_{n}\right), \\
i=n & \text { for }\left(C_{n}\right), \\
i=1, n-1, n & \text { for }\left(D_{n}\right), \\
i=1,6 & \text { for }\left(E_{6}\right), \\
i=1 & \text { for }\left(E_{7}\right) .
\end{array}
$$

List 6.2.2.

Example 6.2.3. Let $\mathfrak{g}_{\mathbb{C}}=\mathfrak{g l}(n, \mathbb{C})$ and $\lambda=\left(\lambda_{1}, \ldots, \lambda_{n}\right) \in \mathbb{Z}^{n}, \lambda_{1} \geq$ $\lambda_{2} \geq \cdots \geq \lambda_{n}$. Then $\pi_{\lambda}$ is a pan representation if and only if

$$
\lambda_{1}=\cdots=\lambda_{p} \geq \lambda_{p+1}=\cdots=\lambda_{n}
$$

for some $p(1 \leq p \leq n-1)$. Then, $\left(\mathfrak{g}_{\mathbb{C}}\right)_{\lambda}$ is given by $\mathfrak{g l}(p, \mathbb{C})+\mathfrak{g l}(n-p, \mathbb{C})$. The $k$ th symmetric tensor representation $S^{k}\left(\mathbb{C}^{n}\right)(k \in \mathbb{N})$ and the $k$ th exterior representation $\Lambda^{k}\left(\mathbb{C}^{n}\right)(0 \leq k \leq n)$ are very special examples of pan representations because the highest weight is given by $(k, 0, \ldots, 0)$ and $(1,1, \ldots, 1,0, \ldots, 0)$, respectively.

Okada [53] studied a specific class of irreducible finite dimensional representations of classical Lie algebras, which he refers as "rectangular-shaped representations". This notion coincides with "pan representations" for the type $\left(A_{n}\right),\left(B_{n}\right)$, and $\left(C_{n}\right)$. For the type $\left(D_{n}\right)$, we note that the pan representations $\pi_{k \omega_{1}}(k \in \mathbb{N})$ do not belong to his rectangular representations.

As we shall see in Section 8, irreducible unitary highest weight representations of scalar type (see Definition 8.1.1) of a non-compact simple Lie group of Hermitian type are an infinite-dimensional version of pan representation.

Here is an application of Theorem 1 to the multiplicity-free problem of tensor product representations.

Theorem 25 ([36, Theorem 7.3]). The tensor product $\pi_{\lambda} \otimes \pi_{\mu}$ of any two pan representations $\pi_{\lambda}, \pi_{\mu}$ decomposes with multiplicity free.

In other words, we have:

Theorem 25' (multiplicity-free tensor product: finite dimensionalcase). The tensor product representation $\pi_{k \omega_{i}} \otimes \pi_{l \omega_{j}}$ of a simple Lie algebra decomposes with multiplicity free for any non-negative integers $k$ and $l$ if $i$ and $j$ are in the List 6.2.2. 
For example, some of classical cases have been studied by case-by-case combinatorial argument (see Okada [53], and references therein). A part of Theorem 26 (namely, in the case where $\mathfrak{h}_{\mathbb{C}}$ is the Levi part of some maximal parabolic subalgebra of $\mathfrak{g}_{\mathbb{C}}$ ) was obtained in Littelmann [49] by a different method, that is, by showing the existence of an open orbit of a Borel subgroup.

Example 6.2.4. If $\lambda, \mu \in \mathbb{Z}^{n}$ is of the form

$$
\lambda=(\underbrace{a, \ldots, a}_{p}, \underbrace{b, \ldots, b}_{n-p}), \quad \mu=(\underbrace{x, \ldots, x}_{k}, \underbrace{y, \ldots, y}_{n-k})
$$

for some $p, k, a \geq b$ and $x \geq y$, then the tensor product representation $\pi_{\lambda} \otimes \pi_{\mu}$ of $\mathfrak{g l}(n, \mathbb{C})$ decomposes with multiplicity free.

The tensor product representation can be regarded as a special case of restrictions, namely, it is the restriction of a representation of the Lie algebra $\mathfrak{g}_{\mathbb{C}} \oplus \mathfrak{g}_{\mathbb{C}}$ with respect to its diagonal subalgebra

$$
\operatorname{diag} \mathfrak{g}_{\mathbb{C}}:=\left\{(X, X): X \in \mathfrak{g}_{\mathbb{C}}\right\} .
$$

In light that $\left(\mathfrak{g}_{\mathbb{C}} \oplus \mathfrak{g}_{\mathbb{C}}, \operatorname{diag} \mathfrak{g}_{\mathbb{C}}\right)$ forms a symmetric pair, the following theorem gives a generalization of Theorem 25 .

Theorem 26 (multiplicity-free restriction for symmetric pairs). Let $\left(\mathfrak{g}_{\mathbb{C}}, \mathfrak{h}_{\mathbb{C}}\right)$ be a complex reductive symmetric pair. Then, for any irreducible pan representation $\pi_{\lambda}$ (see Definition 6.2.1), the restriction $\left.\pi_{\lambda}\right|_{\mathfrak{h}_{\mathbb{C}}}$ decomposes with multiplicity free.

As far as the author is aware, Theorems 26 in this generality is not known, although some particular cases were previously known by different argument such as the open Borel orbit criterion and combinatorial techniques. Our proof of Theorems 25 and 26 is based on Theorem 1, for which we shall report in another paper. There, Corollary 13 (1) and Theorem 12 (visible actions on Hermitian symmetric spaces) play a key role in geometry. One of the advantages of our approach is that it brings analogous multiplicity-free theorems of infinite dimensional representations as well (see Theorems 34 and 40).

Example 6.2.5. Suppose $\pi_{\lambda}$ is an irreducible representation of $\mathfrak{g}_{\mathbb{C}}=$ $\mathfrak{g l}(n, \mathbb{C})$ with highest weight

$$
\lambda=(\underbrace{a, \ldots, a}_{p}, \underbrace{b, \ldots, b}_{n-p}) \in \mathbb{Z}^{n} \quad(a \geq b) .
$$


Then, $\pi_{\lambda}$ decomposes into a multiplicity-free sum of irreducible representations of $\mathfrak{h}_{\mathbb{C}}$ when restricted to the subalgebra $\mathfrak{h}_{\mathbb{C}}$ of the following form:

$$
\mathfrak{h}_{\mathbb{C}}= \begin{cases}\mathfrak{g l}(k, \mathbb{C}) \oplus \mathfrak{g l}(n-k, \mathbb{C}) & (1 \leq k \leq n), \\ \mathfrak{o}(n, \mathbb{C}), & (n: \text { even })\end{cases}
$$

Explicit branching laws for some of classical cases are known in recent years. For example, the branching laws of the restriction $\left.\pi_{\lambda}\right|_{\mathfrak{h}_{\mathbb{C}}}$ are obtained by Okada in the following special cases:

$$
\begin{aligned}
& p=k, \quad \mathfrak{h}_{\mathbb{C}}=\mathfrak{g l}(k, \mathbb{C}) \oplus \mathfrak{g l}(n-k, \mathbb{C}), \quad \text { [53, Theorem 2.1], } \\
& p=\left[\frac{n}{2}\right], \quad \mathfrak{h}_{\mathbb{C}}=\mathfrak{o}(n, \mathbb{C}) \quad \text { or } \quad \mathfrak{s p}\left(\frac{n}{2}, \mathbb{C}\right), \quad[53, \text { Theorem 2.6]. }
\end{aligned}
$$

As one knows a priori by Theorem 26, Okada's formulas are all multiplicityfree. For some of exceptional symmetric pairs $\left(\mathfrak{g}_{\mathbb{C}}, \mathfrak{h}_{\mathbb{C}}\right)$ such as $\left(\mathfrak{e}_{6}, \mathfrak{f}_{4}\right)$, Alikawa [1] has computed multiplicity-free branching laws of pan representations.

\section{§6.3. Multiplicity-free tensor product representations of $\mathfrak{g l}(n, \mathbb{C})$}

We end this section with multiplicity-free tensor product representations of $\mathfrak{g l}(n, \mathbb{C})$. We have already seen some of such examples in Example 6.2.4. The main machinery there was Theorem 1 . We recall that Theorem 1 is strengthened by Theorem 2. Then, it turns out that Theorem 2 combined with the Grassmann geometry (see Theorem 16) yields the list of all multiplicity-free tensor product representations of $\mathfrak{g l}(n, \mathbb{C})$ as follows:

Suppose that $\lambda$ is of the form

$$
\lambda=(\underbrace{a, \ldots, a}_{p}, \underbrace{b, \ldots, b}_{q}) \in \mathbb{Z}^{n}
$$

for some $p, q, a$ and $b$ such that $p+q=n$ and $a \geq b$.

Theorem 27 (multiplicity-free tensor product of $G L_{n},[38,66]$ ). The tensor product representation $\pi_{\lambda} \otimes \pi_{\nu}$ decomposes with multiplicity free in the following cases (divided according to the condition on $\lambda$ ):

Case 1) $\min (a-b, p, q)=1$ (and $\nu$ is arbitrary).

Case 2) $\min (a-b, p, q)=2$, and $\nu$ is of the form 


$$
(\underbrace{x, \ldots, x}_{n_{1}}, \underbrace{y, \ldots, y}_{n_{2}}, \underbrace{z, \ldots, z}_{n_{3}})
$$

where $x \geq y \geq z$ and $n_{1}+n_{2}+n_{3}=n$.

Case 3) $\min (a-b, p, q) \geq 3$, and $\nu$ is of the form (6.2) with $\min (x-y, y-$ $\left.z, n_{1}, n_{2}, n_{3}\right)=1$.

Remark 6.3.1. Stembridge [66] gave a case-by-case combinatorial proof of Theorem 27. He also proved that the above list exhausts all the multiplicityfree cases up to switch of factor. Our proof of Theorem 27 (see [38, Theorem 3.6]) is geometric, and is completely different from [66]. Some of explicit decomposition formulas have been obtained recently; for example, see Krattenthaler [46] in the case $n_{3}=1$.

\section{§7. Multiplicity-Free Representations - Compact Examples}

In Section 6, we have presented a number of multiplicity-free theorems for finite dimensional representations of reductive Lie algebras $\mathfrak{g}_{\mathbb{C}}$. By the Weyl unitary trick, they can be interpreted also as those of compact Lie groups $G$.

However, there are some other "intrinsic" representations of compact Lie groups, some of which are multiplicity-free. The Peter-Weyl theorem is a typical example. This section collects a few more examples of multiplicity-free representations of this kind. A large part of the results in this section is known, yet we shall see that all of them are covered again by our multiplicity-free theorem (Theorem 2).

\section{$\S 7.1$ Regular representation on symmetric spaces - scalar case}

Let $K$ be a compact Lie group. First of all, let us recall the Peter-Weyl theorem, which gives a decomposition of the regular representation on $L^{2}(K)$ as a sum of irreducible representations of the direct product group $K \times K$ :

$$
L^{2}(K) \simeq \sum_{\mu \in \widehat{K}}^{\oplus} \mu \nabla \mu^{*} .
$$

Among all, we pin down the following property:

Fact 28 (Peter-Weyl). The regular representation $L^{2}(K)$ is multiplicity-free as a representation of $G:=K \times K$. 
Via the $(K \times K)$-equivariant isomorphism $K \simeq(K \times K) / \operatorname{diag} K($ see $(5.1))$, Fact 28 can be regarded as a special case of the following statement:

Fact 29 (É. Cartan). The regular representation $L^{2}(G / K)$ on a compact symmetric space $G / K$ is multiplicity-free as a representation of $G$.

This is a compact version of Corollary 22 in Section 5. This fact was established by É. Cartan [7, Sect. 17] by going through all cases, and was later by I. M. Gelfand [13] by using a more general method of the Hecke algebra. Below we shall give a new proof of this fact (and its generalization) by using our general machinery. An explicit decomposition formula for $L^{2}(G / K)$ is known as the Cartan-Helgason theorem (see [73, §3.3.1]) for a connected $G$.

From our viewpoint, the geometric background of Facts 28 and 29 is the visibility of the $(K \times K)$-action on $K_{\mathbb{C}}$ (Theorem 10) and the $G$-action on $G_{\mathbb{C}} / K_{\mathbb{C}}$ (Theorem 11). We shall explain this viewpoint in the process of generalizing Fact 29 (see Theorem 30 below).

\section{§7.2. Regular representation on symmetric spaces - vector bundle case}

This subsection discusses a generalization

$$
\text { Fact } 28 \Rightarrow \text { Fact } 29 \Rightarrow \text { Theorem } 30
$$

by replacing "functions" by "sections" for vector bundles. Again the main machinery for this will be the complex geometric approach given in Theorem 2 .

Let $(G, K)$ be a compact symmetric pair, and we fix a (finite dimensional, unitary) representation $\mu$ of $K$. Then we form a $G$-equivariant Hermitian vector bundle $G \times_{K} \mu \rightarrow G / K$, and define a unitary representation of $G$ on the Hilbert space $L^{2}\left(G \times_{K} \mu\right)$ consisting of $L^{2}$-sections.

Unlike the scalar case $L^{2}(G / K)$, the unitary representation $L^{2}\left(G \times_{K} \mu\right)$ is not always multiplicity-free even though $(G, K)$ is a compact symmetric pair. Let us apply Theorem 2 to find the condition on $\mu$ for which $L^{2}\left(G \times_{K} \mu\right)$ becomes multiplicity-free as a representation of $G$.

We write $\mathfrak{g}=\mathfrak{k}+\mathfrak{p}$ for the Cartan decomposition of the Lie algebra $\mathfrak{g}$ of $G$. Take a maximal abelian subspace $\mathfrak{a}$ of $\mathfrak{p}$, and we set

$$
M:=\{k \in K: \operatorname{Ad}(k) X=X \quad \text { for any } X \in \mathfrak{a}\} .
$$

Then, we have: 
Theorem 30 (cf. Deitmar [8]). Let $(G, K)$ be a compact symmetric pair, and $\mu$ a finite dimensional representation of $K$. Then the regular representation of $G$ on $L^{2}\left(G \times_{K} \mu\right)$ is multiplicity-free if the restriction $\left.\mu\right|_{M}$ is multiplicity-free as a representation of $M$.

Proof. Our proof is built on the visible action of $G$ on the complexified symmetric space $G_{\mathbb{C}} / K_{\mathbb{C}}$ (see Theorem 11 in Section 5 ).

What we need is to prove $m_{\pi} \leq 1$ for all $\pi \in \widehat{G}$, where $m_{\pi}$ is the multiplicity defined by $m_{\pi}:=\operatorname{dim} \operatorname{Hom}_{G}\left(\pi, L^{2}\left(G \times_{K} \mu\right)\right)$.

We extend $\mu$ to a holomorphic representation of $K_{\mathbb{C}}$ (and still use the same notation $\mu$ ). Then, we have naturally a bundle map

$$
\begin{array}{cccc}
G \times_{K} \mu & \hookrightarrow & G_{\mathbb{C}} \times_{K_{\mathbb{C}}} \mu \\
\downarrow & & \downarrow \\
G / K & \hookrightarrow & G_{\mathbb{C}} / K_{\mathbb{C}}
\end{array}
$$

which induces an injective $G$-homomorphism

$$
\mathcal{O}\left(G_{\mathbb{C}} \times_{K_{\mathbb{C}}} \mu\right) \hookrightarrow L^{2}\left(G \times_{K} \mu\right)
$$

by the unicity theorem of holomorphic functions.

Since any matrix coefficient of a finite dimensional representation of $G$ extends holomorphically to $G_{\mathbb{C}}$, the image of the homomorphism (7.2) contains an algebraic direct sum $\bigoplus_{\pi \in \widehat{G}} m_{\pi} \pi$, which is a dense subspace of $L^{2}\left(G \times_{K} \mu\right)$.

Take any $\pi \in \widehat{G}$, and we realize the multiple $m_{\pi} \pi \simeq \pi \oplus \cdots \oplus \pi$ of the unitary representation $\pi$ of $G$ in $\mathcal{O}\left(G_{\mathbb{C}} \times_{K_{\mathbb{C}}} \mu\right)$. As in the proof of Theorem 11, we set $B:=\exp (\sqrt{-1} \mathfrak{a})$. We note that the subgroup $M$ (see (7.1) for the definition) coincides with $Z_{G \cap K_{\mathbb{C}}}(B)=Z_{K}(B)$, the centralizer of $B$ in $K$. Then, applying Theorem 2 to the setting where $(P, H, K, \mu, B)$ with the notation of Theorem 2 is $\left(G_{\mathbb{C}}, G, K_{\mathbb{C}}, \mu, B\right)$ with the notation here, we conclude that $m_{\pi}=1$. Hence Theorem 30 has been proved.

Example 7.2.1. The assumption on $\mu$ in Theorem 30 is obviously satisfied if $\operatorname{dim} \mu=1$, especially if $\mu$ is the trivial one dimensional representation. Then, the above theorem is nothing but Fact 29 .

Example 7.2.2. The assumption in Theorem 30 is satisfied for any $\mu \in \widehat{K}$ if $(G, K)=(U(n), U(1) \times U(n-1))$ or $(O(n), O(n-1))$. This will be discussed in Facts 31 and 32.

Example 7.2.3 (Restriction $U(n) \downarrow O(n))$. The assumption in Theorem 30 is satisfied for $\mu=\Lambda^{k}\left(\mathbb{C}^{n}\right)(0 \leq k \leq n)$ if $(G, K)=(U(n), O(n))$. 
Hence, $L^{2}\left(U(n) \times_{O(n)} \Lambda^{k}\left(\mathbb{C}^{n}\right)\right)$ is multiplicity-free as a $U(n)$-module for any $k$. By the Frobenius reciprocity, this is also equivalent to the following statement:

$$
\operatorname{dim} \operatorname{Hom}_{O(n)}\left(\Lambda^{k}\left(\mathbb{C}^{n}\right),\left.\pi\right|_{O(n)}\right) \leq 1
$$

for any $\pi \in \widehat{U(n)}$ and for any $k(0 \leq k \leq n)$.

Proof of Example 7.2.3. We observe that $B \simeq \mathbb{R}^{n}$ and $M \simeq(\mathbb{Z} / 2 \mathbb{Z})^{n}$ if $(G, K)=(U(n), O(n))$. Then, since the exterior representation $\Lambda^{k}\left(\mathbb{C}^{n}\right)$ is multiplicity-free when restricted to $M$, Example 7.2.3 is led by Theorem 30 .

\section{$\S 7.3$. Some finite dimensional applications}

We end up this section with some applications of Theorem 30 to branching laws. It is very well-known that the restriction from $U(n)$ to $U(n-1)$, and from $O(n)$ to $O(n-1)$ is multiplicity-free. One way to see multiplicity-freeness is of course to observe the explicit branching laws (e.g. [77]). An alternative approach (e.g. [70]) is to show that a Borel subgroup of $H_{\mathbb{C}}$ acts on the flag variety of $G_{\mathbb{C}}$ with an open orbit if

$$
\begin{gathered}
\left(G_{\mathbb{C}}, H_{\mathbb{C}}\right)=(G L(n, \mathbb{C}), G L(n-1, \mathbb{C})) \\
\text { or } \quad(O(n, \mathbb{C}), O(n-1, \mathbb{C})) .
\end{gathered}
$$

As an alternative approach from our viewpoint on visible actions, we shall show that Theorem 30 implies these classical results:

Fact $31(O(n) \downarrow O(n-1)) . \quad$ For any $\pi \in \widehat{O(n)}$, the restriction $\left.\pi\right|_{O(n-1)}$ decomposes with multiplicity free.

Proof of Fact 31. By the Frobenius reciprocity, the assertion is equivalent to:

$\left(P_{n}\right) \quad$ Let $(G, K)=(O(n), O(n-1))$. Then $L^{2}\left(G \times_{K} \mu\right)$ is multiplicity-free as a representation of $G$ for any $\mu \in \widehat{K}$.

The statement $\left(P_{n}\right)$ is obvious if $n=1$. Suppose $n \geq 2$. Then, the restriction $\left.\mu\right|_{M}$ is multiplicity-free for any $\mu \in \widehat{K}$ by the inductive assumption $\left(P_{n-1}\right)$ in light that $(K, M) \simeq(O(n-1), O(n-2))$. Hence, $\left(P_{n}\right)$ holds by Theorem 30. Therefore $\left(P_{n}\right)$ is proved for any $n$ by induction.

A similar proof also works for the restriction $U(n) \downarrow U(n-1)$ : 
Fact $32(U(n) \downarrow U(n-1))$. For any $\pi \in \widehat{U(n)}$, the restriction $\left.\pi\right|_{U(n-1)}$ decomposes with multiplicity free.

As a third method, we should mention that Fact 32 can be shown also directly from the visibility of the action of $U(1) \times U(n-1)$ on the full flag variety $\mathcal{B}\left(\mathbb{C}^{n}\right)$ as explained Example 5.1.4. (This was the approach taken in [38, Example 2.4].)

\section{§8. Multiplicity-Free Representations - Infinite Dimensional Examples}

So far, we have discussed finite dimensional examples. This section provides a number of multiplicity-free theorems for infinite dimensional representations. Again, Theorem 1 (or Theorem 2) serves as a main machinery here. One of the advantages of our approach is that we can treat the infinite dimensional case in a parallel fashion to the finite dimensional case (see Section 6). It should be noted that some of branching laws here contain continuous spectrum, which occurs with multiplicity free. Typical cases may be compared in the following correspondences:

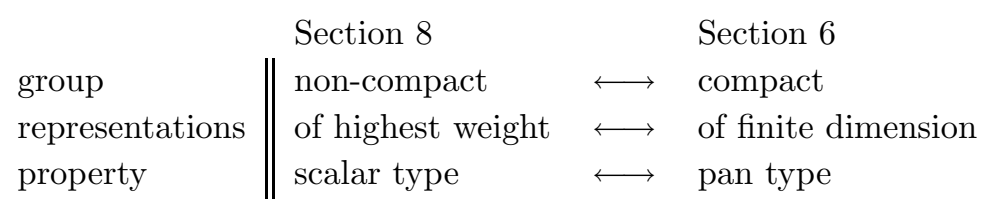

\section{§8.1. Unitary highest weight representations}

We review some basic notion regarding highest weight modules. Let $G$ be a non-compact simple Lie group, $\theta$ a Cartan involution, and $K:=G^{\theta}$. We write $\mathfrak{g}=\mathfrak{k}+\mathfrak{p}$ for the Cartan decomposition of the Lie algebra $\mathfrak{g}$ of $G$, corresponding to the Cartan involution $\theta$.

We say $G$ is of Hermitian type if the center $\mathfrak{c}(\mathfrak{k})$ of $\mathfrak{k}$ is non-trivial. Then, $\operatorname{dim} \mathfrak{c}(\mathfrak{k})=1$ and there exists a characteristic element $Z \in \mathfrak{c}(\mathfrak{k})$ so that

$$
\mathfrak{g}_{\mathbb{C}}:=\mathfrak{g} \otimes \mathbb{C}=\mathfrak{k}_{\mathbb{C}} \oplus \mathfrak{p}^{+} \oplus \mathfrak{p}^{-}
$$

is the eigenspace decomposition of $\operatorname{ad}(Z)$ with eigenvalues $0, \sqrt{-1}$ and $-\sqrt{-1}$, respectively. Then, the homogeneous space $G / K$ becomes a Hermitian symmetric space of non-compact type. 
Here is a list of Lie algebras corresponding to non-compact simple Lie groups of Hermitian type:

$$
\mathfrak{s u}(p, q), \mathfrak{s p}(m, \mathbb{R}), \mathfrak{s o}(n, 2), \mathfrak{s o}^{*}(2 n), \mathfrak{e}_{6(-14)}, \mathfrak{e}_{7(-25)},
$$

where $p, q, m \geq 1$ and $n \geq 3$.

Suppose that $(\pi, \mathcal{H})$ is an irreducible unitary representation of $G$, and $\mathcal{H}_{K}$ is the underlying $\left(\mathfrak{g}_{\mathbb{C}}, K\right)$-module. We define a subspace of $\mathcal{H}_{K}$ by

$$
\mathcal{H}_{K}^{\mathfrak{p}^{+}}:=\left\{v \in \mathcal{H}_{K}: d \pi(Y) v=0 \text { for any } Y \in \mathfrak{p}^{+}\right\}
$$

Then, it is known that $\mathcal{H}_{K}^{\mathfrak{p}^{+}}$is either zero or irreducible as a representation of $K$.

Definition 8.1.1. We say $(\pi, \mathcal{H})$ is an irreducible unitary highest weight representation if $\mathcal{H}_{K}^{\mathfrak{p}^{+}} \neq\{0\}$. Furthermore, $\pi$ is of scalar type if $\operatorname{dim} \mathcal{H}_{K}^{\mathfrak{p}^{+}}=1$.

An irreducible (unitary) highest weight representation $(\pi, \mathcal{H})$ of $G$ is determined uniquely by the $K$-module structure on $\mathcal{H}_{K}^{\mathfrak{p}^{+}}$. We fix a maximal abelian subspace $\mathfrak{t}$ of $\mathfrak{k}$, and a positive system $\Delta^{+}(\mathfrak{k}, \mathfrak{t})$. The representation $(\pi, \mathcal{H})$ will be denoted by $\pi^{G}(\mu)$ if $\mu \in \sqrt{-1} \mathfrak{t}^{*}$ is a highest weight of the $K$-module $\mathcal{H}_{K}^{\mathfrak{p}^{+}}$ with respect to the positive system $\Delta^{+}(\mathfrak{k}, \mathfrak{t})$.

An irreducible unitary highest weight representation $\pi$ is called a (relative) holomorphic discrete series representation for $G$, if any matrix coefficient of $\pi$ is square integrable over $G$ modulo its center. Lowest weight representations and anti-holomorphic discrete series representations are defined similarly with $\mathfrak{p}^{+}$replaced by $\mathfrak{p}^{-}$.

The classification of irreducible unitary highest weight representations was accomplished in Enright-Howe-Wallach [10] and Jakobsen [25] (see also [11]). There exist infinitely many unitary highest weight representations of scalar type for any non-compact simple Lie group of Hermitian type.

\section{§8.2. Restriction to a unipotent subgroup $N$}

Let $G$ be a non-compact simple Lie group of Hermitian type, and $N$ a maximal unipotent subgroup of $G$. Then, our abstract multiplicity-free theorem (Theorem 1) combined with the (strong) visibility of the action (Theorem 14) leads us to the following multiplicity-free theorem: 
Theorem 33 (restriction to a unipotent subgroup). If $\pi$ is an irreducible unitary highest weight representation of $G$ of scalar type, then the restriction $\left.\pi\right|_{N}$ is multiplicity-free.

Example 8.2.1 (Hardy space). The (classical) Hardy space is the Fourier image of $L^{2}$-functions supported on the positive half line. A group theoretic aspect of this is the fact that the limit of holomorphic discrete series representation $\pi$ of $G=S L(2, \mathbb{R})$ is decomposed into the direct integral of $e^{i x \xi}$ $(\xi \geq 0)$ with multiplicity one, when restricted to a maximal unipotent subgroup $N \simeq \mathbb{R}$.

\section{$\S 8.3$. Restriction to a symmetric subgroup}

We keep our setting that $G$ is a non-compact simple Lie group of Hermitian type. Here is a main multiplicity-free theorem in this section. It may be regarded as an infinite dimensional version of Theorem 26 .

Theorem 34 (multiplicity-free restriction for symmetric pairs, [32]). Suppose $\pi$ is an irreducible unitary highest weight representation of $G$ of scalar type. Then, the restriction $\left.\pi\right|_{H}$ decomposes with multiplicity free for any subgroup $H$ such that $(G, H)$ is a semisimple symmetric pair.

Here, we should note that the subgroup $H$ is not necessarily compact. Accordingly, the restriction $\left.\pi\right|_{H}$ may contain continuous spectrum (see Fact 35 and Theorem 36 below for more details).

We refer to [36, Theorem A] for a proof of Theorem 34 based on our machinery, i.e., Theorem 1. For some classical cases, one could give an alternative proof of Theorem 34 (and also Theorem 39 below) by case-by-case argument, by using the idea of see-saw dual pairs ([19]). However, our approach to Theorem 34 is simple and geometric. It connects branching problems $\left.\pi\right|_{H}$ with the geometry of the $H$-action on the Hermitian symmetric space, which is (strongly) visible (see Theorem 12).

Suppose that $(G, H)$ is a symmetric pair defined by an involutive automorphism $\tau$ of $G$, namely, $\left(G^{\tau}\right)_{0} \subset H \subset G^{\tau}$. We may and do take a Cartan involution $\theta$ of $G$ such that $\tau \theta=\theta \tau$. Then, $\tau$ stabilizes $K\left(=G^{\theta}\right)$, its Lie algebra $\mathfrak{k}$, and also the center $\mathfrak{c}(\mathfrak{k})$ of $\mathfrak{k}$. Since $\mathfrak{c}(\mathfrak{k})$ is of one dimension, $\tau$ acts on $\mathfrak{c}(\mathfrak{k})$ by either +1 or -1 because $\tau^{2}=$ id.

Definition 8.3.1. We say a symmetric pair $(G, H)$ is of holomorphic type (respectively, of anti-holomorphic type) if $\tau$ acts on $\mathfrak{c}(\mathfrak{k})$ as the scalar +1 (respectively, -1 ). 
Then, the natural embedding $H / H \cap K \hookrightarrow G / K$ is holomorphic if $(G, H)$ is of holomorphic type, while it is totally real if $(G, H)$ is of anti-holomorphic type.

Here is a sharp contrast on the existence of continuous spectrum in the branching law:

Fact 35 ([33, 34]). Suppose $(G, H)$ is a symmetric pair of holomorphic type and that $\pi$ is a unitary highest weight representation of $G$. Then the restriction $\left.\pi\right|_{H}$ decomposes discretely.

Theorem 36. Suppose $(G, H)$ is a symmetric pair of anti-holomorphic type, and that $\pi$ is a holomorphic discrete series representation of $G$.

1) The restriction $\left.\pi\right|_{H}$ always contains continuous spectrum.

2) There are at most a finite number of discrete spectrum in the branching law of the restriction $\left.\pi\right|_{H}$.

3) If $\pi$ is furthermore of scalar type, then there is no discrete spectrum in $\left.\pi\right|_{H}$.

Theorem 36 is a consequence of the result of Howe, Repka, Ólafsson and Ørsted $([19,59,54])$ that the restriction $\left.\pi\right|_{H}$ is unitarily equivalent to $L^{2}\left(H \times{ }_{H \cap K} \mu\right)$ if $\pi=\pi^{G}(\mu)$. We should note that the restriction map from $\left(L^{2} \cap \mathcal{O}\right)\left(G \times_{K} \mu\right)$ to $L^{2}\left(H \times_{H \cap K} \mu\right)$ is not unitary but has a dense image. For $\pi$ of scalar type, i.e. $\operatorname{dim} \mu=1$, explicit Plancherel formula for the restriction $\left.\pi\right|_{H}$ has been recently studied by Neretin [52], etc.

We end this subsection with two exclusive examples of Theorem 34 according to holomorphic and anti-holomorphic type (Definition 8.3.1) by assuming furthermore that $\pi$ is a holomorphic discrete series representation.

Example 8.3.2. The Hua-Kostant-Schmid formula gives the branching law of $\left.\pi\right|_{K}$. It is multiplicity-free, and is a special case of Theorem 34 and Fact 35 .

Example 8.3.3. $\quad$ Suppose $(G, H)$ is of anti-holomorphic type (see Definition 8.3.1). Then the restriction $\left.\pi\right|_{H}$ is an example of Theorems 34 and 36, and gives rise to a "canonical representation" in the sense of Gelfand-GraevVershik, which is unitarily equivalent to $L^{2}(H / H \cap K)$ as an abstract unitary representation. 


\section{$\S 8.4$. Boundedness and uniform boundedness of multiplicities}

This subsection reviews known facts on the multiplicity in the branching laws $\left.\pi\right|_{H}$ with respect to a reductive symmetric pair $(G, H)$ without any assumption on $\pi \in \widehat{G}$, and examines the status of Theorem 34 from wider perspectives.

For $\tau \in \widehat{H}$ and $\pi \in \widehat{G}$, we set

$$
m_{\pi}(\tau):=\operatorname{dim} \operatorname{Hom}_{H}\left(\tau,\left.\pi\right|_{H}\right),
$$

the dimension of the space of continuous $H$-homomorphisms.

Suppose $(G, H)$ is a reductive symmetric pair. In general, $m_{\pi}(\tau)$ can be infinity. Even finite multiplicities and infinite multiplicities can stand together in the same branching law as below:

Example 8.4.1 (see [35, Example 5.5]). Let $(G, H)=(S O(5, \mathbb{C})$, $S O(3,2))$, and $\pi$ be a (certain) principal series representation of $G$. Then,

1) $\left.\pi\right|_{H}$ contains both continuous and discrete spectrum.

As for discrete spectrum, the following statements hold:

2) For some $\sigma_{1} \in \widehat{H}, \operatorname{dim} \operatorname{Hom}_{H}\left(\sigma_{1},\left.\pi\right|_{H}\right)=\infty$.

3) For some $\sigma_{2} \in \widehat{H}, 0<\operatorname{dim} \operatorname{Hom}_{H}\left(\sigma_{2},\left.\pi\right|_{H}\right)<\infty$.

However, if the restriction decomposes discretely, we have a finiteness theorem of multiplicities like Harish-Chandra's admissibility theorem. Here, we just mention a special case where $\pi$ is a discrete series representation of $G$ (see [35, Conjecture C] and [37, Section 3] for the background and more general results):

Fact 37 (bounded multiplicity theorem, [33, Part III]). If the restriction $\left.\pi\right|_{H}$ is (infinitesimally) discretely decomposable, then $m_{\pi}(\tau)<\infty$ for any $\tau \in \widehat{H}$.

The key machinery of the proof is the associated variety of $\pi$ and the asymptotic behavior of $K$-types. Since this method is out of the scope of this article, we do not go into details here. See [33, Part III] and [39, Section 5].

We should remark that $m_{\pi}(\tau)$ is bounded but not always uniformly bounded in Fact 37. This can happen even in the case $H=K$ (a maximal compact subgroup of $G$ ). 
Example 8.4.2. $\quad$ Let $(G, H)=(S p(2, \mathbb{R}), U(2))$, and $\pi$ be a non-holomorphic discrete series representation. Then we have

1) $m_{\pi}(\tau)<\infty$ for any $\pi \in \widehat{H}$.

2) $\sup _{\tau \in \widehat{H}} m_{\pi}(\tau)=\infty$.

Thus, the following uniform boundedness theorem is noteworthy for any highest weight representations $\pi$ (not necessarily of scalar type).

Theorem 38 (uniform boundedness theorem, [36, Theorem B]). Suppose $(G, H)$ is a symmetric pair of holomorphic type, and $\pi$ is an irreducible unitary highest weight representation of $G$. Then, we have

$$
\sup _{\tau \in \widehat{H}} m_{\pi}(\tau)<\infty
$$

Sketch of Proof. Use Theorem 34 and tensoring argument.

\section{§8.5. A generalization of Hua-Kostant-Schmid formula}

If a representation is known a priori to be multiplicity-free, one may be tempted to find its decomposition formula explicitly.

In this subsection, we present a new explicit decomposition formula of the restriction $\left.\pi\right|_{H}$ which is known a priori to be multiplicity-free by Theorem 34 .

Let $(G, H)$ be of holomorphic type (Definition 8.3.1). We take a Cartan subalgebra $\mathfrak{t}$ of $\mathfrak{k}$ such that $\mathfrak{t}^{\tau}:=\mathfrak{t} \cap \mathfrak{h}$ is also a Cartan subalgebra of $\mathfrak{k} \cap \mathfrak{h}$. We write

$$
\left(\mathfrak{p}^{+}\right)^{-\tau}:=\left\{X \in \mathfrak{p}^{+}: \tau X=-X\right\} .
$$

Let us take a maximal set $\left\{\nu_{1}, \ldots, \nu_{k}\right\}$ of strongly orthogonal roots in $\Delta\left(\left(\mathfrak{p}^{+}\right)^{-\tau}, \mathfrak{t}^{\tau}\right)$. We note that the cardinality $k$ coincides with the real rank (sometimes called the split rank) of the semisimple symmetric space $G / H$.

Theorem 39 (branching law for semisimple symmetric pairs, [32]). Retain the setting as above. Let $\pi^{G}(\mu)$ be a holomorphic discrete series representation of scalar type, and $(G, H)$ a symmetric pair of holomorphic type such that $H$ is connected. Then, the representation $\pi^{G}(\mu)$ decomposes into a discrete, multiplicity-free sum of irreducible representations of $H$ as follows:

$$
\left.\pi^{G}(\mu)\right|_{H} \simeq \sum_{\substack{a_{1} \geq \cdots \geq a_{k} \geq 0 \\ a_{1}, \ldots, a_{k} \in \mathbb{N}}}^{\oplus} \pi^{H}\left(\left.\mu\right|_{\mathfrak{t}^{\tau}}-\sum_{j=1}^{k} a_{j} \nu_{j}\right) .
$$


We note that the Hermitian symmetric pair $(G, K)$ itself is a holomorphic type. In this special setting where $H=K$, all representations in the right side of (8.2) are finite dimensional. In this case, the branching law (8.2) is due to Hua (classical), Kostant (unpublished), and Schmid [62]. Johnson [27] gave an alternative proof, again in the compact $H$ case. In the general case with $H$ noncompact the formula was given by the author [32] with a sketch of proof.

\section{§8.6. Multiplicity-free tensor product representations}

Parallel to the multiplicity-free result (Theorem 34) for the reductive symmetric pair $(G, H)$, we can also prove a multiplicity-free theorem for the tensor product representations:

Theorem 40 (multiplicity-free tensor product: infinite dimensional case; $[32,36])$.

1) If $\pi_{1}$ and $\pi_{2}$ are irreducible unitary highest weight representations of scalar type, then the tensor product representation $\pi_{1} \widehat{\otimes} \pi_{2}$ decomposes discretely into a sum of irreducible representations with multiplicity free.

2) If $\pi_{1}$ (respectively, $\pi_{2}$ ) is an irreducible unitary highest (respectively, lowest) weight representation, then the tensor product $\pi_{1} \widehat{\otimes} \pi_{2}$ is still multiplicityfree.

Here, $\widehat{\otimes}$ denotes the Hilbert completion of an algebraic tensor product.

Remark 8.6.1.

1) We notice that the decomposition formula in the case (2) contains continuous spectrum in general. In particular, this is the case if $\pi_{1}$ is a holomorphic discrete series representation and $\pi_{2}$ is an anti-holomorphic discrete series representation.

2) The underlying geometry which we need in the proof is the (strong) visibility of the diagonal action of $G$ on the direct product manifold $G / K \times G / K$ (see Corollary 13).

3) Theorem 40 can be regarded as an infinite dimensional version of the multiplicity-free result (Theorem 25) for finite dimensional representations. 


\section{§9. The Orbit Method}

In the spirit of the Kirillov-Kostant orbit method, we may expect that multiplicity-free theorems of representations should be reflected by the geometry of coadjoint orbits.

This section introduces briefly this kind of results obtained jointly with Nasrin [43], which is a counterpart of representation-theoretic results (Theorems 34 and 40).

Let $G$ be a non-compact simple Lie group such that $G / K$ is a Hermitian symmetric space. We shall identify the Lie algebra $\mathfrak{g}$ with its dual $\mathfrak{g}^{*}$, and write $\zeta \in \mathfrak{g}^{*}$ for the corresponding element to the characteristic element $Z \in \mathfrak{c}(\mathfrak{k})$ (see Section 8.1 for the notation). In the spirit of the orbit method, a unitary highest weight module $\pi$ of scalar type may "correspond to" the coadjoint orbit

$$
\mathcal{O}_{\lambda}^{G}:=\operatorname{Ad}^{*}(G)(\lambda \zeta) \subset \sqrt{-1} \mathfrak{g}^{*}
$$

for some $\lambda \in \sqrt{-1} \mathbb{R}$.

Suppose $H$ is a subgroup of $G$, and we write pr $: \mathfrak{g}^{*} \rightarrow \mathfrak{h}^{*}$ for the natural projection of the dual of Lie algebras.

Then the following theorem is a counterpart of the multiplicity-free property of the branching law $\left.\pi\right|_{H}$ (Theorem 34).

Theorem 41 (geometry of coadjoint orbit, [43, Theorem A]). If $(G, H)$ is a symmetric pair, then the intersection $\mathcal{O}_{\lambda}^{G} \cap \operatorname{pr}^{-1}\left(\mathcal{O}^{H}\right)$ is a single $H$-orbit for any $\lambda \in \sqrt{-1} \mathbb{R}$ and for any coadjoint orbit $\mathcal{O}^{H}$ in $\mathfrak{h}^{*}$, whenever it is nonempty.

For a general coadjoint orbit $\mathcal{O}^{G}$ in $\mathfrak{g}^{*}$, there may exist infinitely many $H$-orbits on $\mathcal{O}^{G} \cap \operatorname{pr}^{-1}\left(\mathcal{O}^{H}\right)$. This can happen even when $H=K$. See [43] for such a counterexample in the case $(G, H)=(S L(n, \mathbb{R}), S O(n))(n \geq 3)$.

Applying Theorem 41 to a connected $H$ (by replacing the identity component of $H$ if necessary), we have the following topological observation:

Corollary 42. In the setting of Theorem 41, the intersection $\mathcal{O}_{\lambda}^{G} \cap$ $\operatorname{pr}^{-1}\left(\mathcal{O}^{H}\right)$ is connected for any $\lambda \in \sqrt{-1} \mathbb{R}$ and for any coadjoint orbit $\mathcal{O}^{H}$ in $\mathfrak{h}^{*}$.

Next, let us consider the geometry of coadjoint orbits corresponding to the tensor product representations $\pi_{1} \widehat{\otimes} \pi_{2}$. For simplicity, we treat here the case where $\pi_{1} \simeq \pi_{2}$ or $\pi_{1} \simeq \pi_{2}^{*}$ in Theorem 40 . 
The projection dual to the embedding, $\operatorname{diag} \mathfrak{g} \hookrightarrow \mathfrak{g} \oplus \mathfrak{g}$ is given by

$$
\operatorname{pr}: \mathfrak{g}^{*} \oplus \mathfrak{g}^{*} \rightarrow \mathfrak{g}^{*}, \quad\left(\nu_{1}, \nu_{2}\right) \mapsto \nu_{1}+\nu_{2} .
$$

We write $\mathcal{O}_{\left(\lambda_{1}, \lambda_{2}\right)}^{G \times G}$ for the coadjoint orbit of $G \times G$ which goes through $\left(\lambda_{1} \zeta, \lambda_{2} \zeta\right)$ $\in \sqrt{-1}\left(\mathfrak{g}^{*} \oplus \mathfrak{g}^{*}\right)$ if $\lambda_{1}, \lambda_{2} \in \sqrt{-1} \mathbb{R}$.

Theorem 43 ([43, Theorem B]). The intersection $\mathcal{O}_{(\lambda, \lambda)}^{G \times G} \cap \operatorname{pr}^{-1}\left(\mathcal{O}^{G}\right)$ (respectively, $\left.\mathcal{O}_{(\lambda,-\lambda)}^{G \times G} \cap \operatorname{pr}^{-1}\left(\mathcal{O}^{G}\right)\right)$ is a single $G$-orbit acting diagonally on $\sqrt{-1}\left(\mathfrak{g}^{*} \oplus \mathfrak{g}^{*}\right)$ for any $\lambda \in \sqrt{-1} \mathbb{R}$ and for any coadjoint orbit $\mathcal{O}^{G}$ in $\sqrt{-1} \mathfrak{g}^{*}$, whenever the intersection is non-empty.

The coadjoint orbit $\mathcal{O}_{\lambda}^{G}$ carries naturally a symplectic structure, on which $G$ (and therefore $H$ ) acts by symplectic diffeomorphisms. The projection map pr $: \sqrt{-1} \mathfrak{g}^{*} \rightarrow \sqrt{-1} \mathfrak{h}^{*}$, when restricted to $\mathcal{O}_{\lambda}^{G}$, gives rise to the momentum map

$$
\mu: \mathcal{O}_{\lambda}^{G} \rightarrow \mathfrak{h}^{*}
$$

for the $H$-action on $\mathcal{O}_{\lambda}^{G}$. Then, Theorem 41 (and likewise Theorem 43) asserts that the momentum map separates all $H$-orbits. Relevant results are known by Brion [6], Huckleberry and Wurzbacher [22] for the action of a connected compact Lie group on a projective variety in the context of spherical embedding. It should be noted that the symplectic manifold $\mathcal{O}_{\lambda}^{G}$ is non-compact and that the group $H$ is not necessary compact in Theorem 41.

\section{References}

[1] Alikawa, H., Multiplicity-free branching rules for outer automorphisms of simple Lie algebras, preprint.

[2] Barbasch, D., Spherical unitary dual for split classical groups, preprint.

[3] Ben Saï, S., Weighted Bergman spaces on bounded symmetric domains, Pacific J. Math., 206 (2002), 39-68.

[4] Benson, C. and Ratcliff, G., On multiplicity free actions, Representations of Real and p-adic Groups, E.-C. Tan and C.-B. Zhu eds., Lect. Notes Ser. Inst. Math. Sci. Natl. Univ. Singap., (2004), 221-304.

[5] Bertram, W. and Hilgert, J., Hardy spaces and analytic continuation of Bergman spaces, Bull. Soc. Math. France, 126 (1998), 435-482.

[6] Brion, M., Sur l'image de l'application moment, Lecture Notes in Math., 1296 (1987), 177-192, Springer.

[7] Cartan, É., Sur la détermination d'un système orthogonal complet dans un espace de Riemann symetrique clos, Rend. Circ. Mat. Palermo, 53 (1929), 217-252.

[8] Deitmar, A., Invariant operators on higher K-types, J. Reine Angew. Math., 412 (1990), 97-107.

[9] van Dijk, G. and Hille, S. C., Canonical representations related to hyperbolic spaces, J. Funct. Anal., 147 (1997), 109-139. 
[10] Enright, T., Howe, R. and Wallach, N., A classification of unitary highest weight modules, Representation theory of reductive groups, Progr. in Math., Birkhäuser, 40 (1983), 97-143.

[11] Enright, T. and Joseph, A., An intrinsic classification of unitary highest weight modules, Math. Ann., 288 (1990), 571-594.

[12] Faraut, J. and Thomas, E. G. F., Invariant Hilbert spaces of holomorphic functions, J. Lie Theory, 9 (1999), 383-402.

[13] Gelfand, I. M., Spherical functions on symmetric spaces, Dokl. Akad. Nauk. SSSR, 70 (1950), 5-8.

[14] Gross, B., Some applications of Gelfand pairs to number theory, Bull. Amer. Math. Soc., 24 (1991), 277-301.

[15] Guillemin, V. and Sternberg, S., Multiplicity-free spaces, J. Differential Geom., 19 (1984), 31-56.

[16] Gutkin, E., Coefficients of Clebsch-Gordan for the holomorphic discrete series, Lett. Math. Phys., 3 (1979), 185-192.

[17] Heckman, G. and Schlichtkrull, H., Harmonic Analysis and special functions on symmetric spaces, Perspectives in Mathematics, 16, Academic Press, San Diego, 1994.

[18] Helgason, S., Groups and Geometric Analysis, Academic Press, New York, 1984.

[19] Howe, R., Reciprocity laws in the theory of dual pairs, Representation Theory of Reductive Groups (P. C. Trombi ed.), Progr. in Math., Birkhäuser, 40 (1983), 159-175.

[20] , Perspectives on invariant theory: Schur duality, multiplicity-free actions and beyond, The Schur lectures 1992, Israel Math. Conf. Proc., 8 (1995), 1-182.

[21] Howe, R. E. and Tan, E.-C., Homogeneous functions on light cones: the infinitesimal structure of some degenerate principal series representations, Bull. Amer. Math. Soc., 28 (1993), 1-74.

[22] Huckleberry, A. T. and Wurzbacher, T., Multiplicity-free complex manifolds, Math. Ann., 286 (1990), 261-280.

[23] Jaffee, H., Real forms of Hermitian symmetric spaces, Bull. Amer. Math. Soc., 81 (1975), 456-458.

[24] Jakobsen, H. P., Tensor products, reproducing kernels, and power series, J. Funct. Anal., 31 (1979), 293-305.

[25] - Hermitian symmetric spaces and their unitary highest weight modules, J. Funct. Anal., 52 (1983), 385-412.

[26] Jakobsen, H. P. and Vergne, M., Restrictions and expansions of holomorphic representations, J. Funct. Anal., 34 (1979), 29-53.

[27] Johnson, K., On a ring of invariant polynomials on a Hermitian symmetric space, $J$. Algebra, 67 (1980), 72-81.

[28] $\ldots$, Degenerate principal series and compact groups, Math. Ann., 287 (1990), 703718.

[29] Kac, V., Some remarks on nilpotent orbits, J. Algebra, 64 (1980), 190-213.

[30] Klimyk, A. U. and Gruber, B., Matrix elements for infinitesimal operators of the groups $U(p+q)$ and $U(p, q)$ in a $U(p) \times U(q)$ basis I, II, J. Math. Phys., 20 (1979), 1995-2010, 2011-2013.

[31] Kobayashi, S., Irreducibility of certain unitary representations, J. Math. Soc. Japan, 20 (1968), 638-642.

[32] Kobayashi, T., Multiplicity-free theorem in branching problems of unitary highest weight modules, Proceedings of the Symposium on Representation Theory held at Saga, Kyushu 1997 (ed. K. Mimachi), (1997), 9-17.

[33] Discrete decomposability of the restriction of $A_{\mathfrak{q}}(\lambda)$ with respect to reductive subgroups and its applications, Part I, Invent. Math., 117 (1994), 181-205; Part II, Ann. of Math., 147 (1998), 709-729; Part III, Invent. Math., 131 (1998), 229-256.

[34] , Discrete series representations for the orbit spaces arising from two involutions of real reductive Lie groups, J. Funct. Anal., 152 (1998), 100-135. 
[35] Kobayashi, T., Discretely decomposable restrictions of unitary representations of reductive Lie groups - examples and conjectures, Adv. Stud. Pure Math., Analysis on Homogeneous Spaces and Representation Theory of Lie Groups, Okayama-Kyoto, 26 (2000), 98-126.

[36] symmetric pairs, preprint, UTMS 2000-1.

[37] , Branching problems of unitary representations, Proc. of I.C.M. 2002, at Beijing, vol. 2, Higher Ed. Press, Beijing (2002), 615-627.

[38] , Geometry of multiplicity-free representations of $G L(n)$, visible actions on flag varieties, and triunity, Acta Appl. Math., 81 (2004), 129-146.

[39] , Restrictions of unitary representations of real reductive groups. In: Lie Theory: Unitary Representations and Compactifications on Symmetric Spaces, (eds. P. Anker and B. Ørsted), Progr. Math., Birkhäuser, 229 (2005), 139-207.

[40] , A generalized Cartan decomposition for the double coset space $(U(p) \times$ $U(q)) \backslash U(n) /\left(U\left(n_{1}\right) \times U\left(n_{2}\right) \times U\left(n_{3}\right)\right)$, in preparation.

[41] $\_$Visible actions on symmetric spaces, in preparation.

[42] Multiplicity-free theorem for holomorphic vector bundles, in preparation.

[43] Kobayashi, T. and Nasrin, S., Multiplicity one theorem in the orbit method, Lie Groups and Symmetric Spaces: In memory of F. I. Karpelevich (ed. S. Gindikin), Translation Series 2, American Mathematical Society, 210 (2003), 161-169.

[44] Kobayashi, T. and Ørsted, B., Analysis on the minimal representation of $\mathrm{O}(p, q)$. II. Branching laws, Adv. Math., 180 (2003), 513-550.

[45] Koike, K. and Terada, I., Young-diagrammatic methods for representation theory of the classical groups of type $B_{n}, C_{n}, D_{n}, J$. Algebra, 107 (1987), 466-511.

[46] Krattenthaler, C., Identities for classical group characters of nearly rectangular shape, J. Algebra, 209 (1998), 1-64.

[47] Krötz, B. and Stanton, R., Holomorphic extension of representation (II): geometry and harmonic analysis, to appear in GAFA.

[48] Lee, S.-T. and Loke, H.-Y., Degenerate principal series representations of $U(p, q)$ and 0(p,q), Compositio Math., 132 (2002), 311-348.

[49] Littelmann, P., On spherical double cones, J. Algebra, 166 (1994), 142-157.

[50] Martens, S., The characters of the holomorphic discrete series, Proc. Natl. Acad. Sci. USA, 72 (1975), 3275-3276.

[51] Neeb, K.-H., On some classes of multiplicity free representations, Manuscripta Math., 92 (1997), 389-407.

[52] Neretin, Y. A., Plancherel formula for Berezin deformation of $L^{2}$ on Riemannian symmetric space, J. Funct. Anal., 189 (2002), 336-408.

[53] Okada, S., Applications of minor summation formulas to rectangular-shaped representations of classical groups, J. Algebra, 205 (1998), 337-367.

[54] Ólafsson, G. and Ørsted, B., Generalizations of the Bargmann transform, Lie Theory and its application in physics (Dobrev and Döbner eds.), World Sci., 1996, 3-14.

[55] Ørsted, B. and Zhang, G., Tensor products of analytic continuations of holomorphic discrete series, Canad. J. Math., 49 (1997), 1224-1241.

[56] Panyushev, D., Complexity and nilpotent orbits, Manuscripta Math., 83 (1994), 223-237.

[57] Podestà, F. and Thorbergsson, G., Polar and coisotropic actions on Kähler manifolds, Trans. Amer. Math. Soc., 354 (2002), 1759-1781.

[58] C Coisotropic actions on compact homogeneous Kähler manifolds, Math. Z., 243 (2003), 471-490.

[59] Repka, J., Tensor products of holomorphic discrete series representations, Canad. J. Math., 31 (1979), 836-844.

[60] Richardson, R., Röhrle, G. and Steinberg, R., Parabolic subgroup with abelian unipotent radical, Invent. Math., 110 (1992), 649-671. 
[61] Sato, M. and Kimura, T., A classification of irreducible prehomogeneous vector spaces and their relative invariants, Nagoya Math. J., 65 (1977), 1-155.

[62] Schmid, W., Die Randwerte holomorphe Funktionen auf hermetisch symmetrischen Raumen, Invent. Math., 9 (1969-70), 61-80.

[63] Sekiguchi, H., The Penrose transform for certain non-compact homogeneous manifolds of $U(n, n)$, J. Math. Sci. Univ. Tokyo, 3 (1996), 655-697.

[64] Math. Soc. Japan, 54 (2002), 215-253.

[65] Shimeno, N., The Plancherel formula for spherical functions with a one-dimensional K-type on a simply connected simple Lie group of Hermitian type, J. Fucnt. Anal., 121 (1994), 330-388.

[66] Stembridge, J. R., Multiplicity-free products of Schur functions, Ann. Comb., 5 (2001), 113-121.

[67] Hall-Littlewood symmetric functions and Rogers-Ramanujan identities, Trans. Amer. Math. Soc., 319 (1990), 469-498.

[68] Vinberg, É. B., Complexity of actions of reductive Lie groups, Funct. Anal. Appl., 20 (1986), 1-11.

[69] Vinberg, É. B., Commutative homogeneous spaces and co-isotropic symplectic actions, Russian Math. Surveys, 56 (2001), 1-60.

[70] Vinberg, É. B. and Kimelfeld, B. N., Homogeneous domains on flag manifolds and spherical subgroups of semisimple Lie groups, Funct. Anal. Appl., 12 (1978), 168-174.

[71] Vogan, D. A., Jr., Unitarizability of certain series of representations, Ann. Math., 120 (1984), 141-187.

[72] Wallach, N. R., On the unitarizability of derived functor modules, Invent. Math., 78 (1984), 131-141.

[73] Warner, G., Harmonic Analysis on Semisimple Lie Groups I, II, Springer-Verlag, Berlin, 1972.

[74] Wolf, J., Representations that remain irreducible on parabolic subgroups. Differential geometrical methods in mathematical physics (Proc. Conf., Aix-enProvence/Salamanca, 1979), pp. 129-144, Lecture Notes in Math., 836, Springer, Berlin, 1980.

[75] Xie, J., Restriction of discrete series of $S U(2,1)$ to $S(U(1) \times U(1,1))$, J. Funct. Anal., 122 (1994), 478-518.

[76] Zhang, G., Branching coefficients of holomorphic representations and Segal-Bargmann transform, J. Funct. Anal., 195 (2002), 306-349.

[77] Zhelobenko, D., Compact Lie Groups and their Representations, Transl. Math. Monogr., 40, Amer. Math. Soc., 1973. 